\title{
Observation of a New Excited Beauty Strange Baryon Decaying to $\Xi_{b}^{-} \pi^{+} \pi^{-}$
}

\author{
A. M. Sirunyan et al. ${ }^{*}$ \\ (CMS Collaboration)
}

(Received 8 February 2021; revised 19 March 2021; accepted 23 April 2021; published 25 June 2021)

\begin{abstract}
The $\Xi_{b}^{-} \pi^{+} \pi^{-}$invariant mass spectrum is investigated with an event sample of proton-proton collisions at $\sqrt{s}=13 \mathrm{TeV}$, collected by the CMS experiment at the LHC in 2016-2018 and corresponding to an integrated luminosity of $140 \mathrm{fb}^{-1}$. The ground state $\Xi_{b}^{-}$is reconstructed via its decays to $J / \psi \Xi^{-}$and $J / \psi \Lambda K^{-}$. A narrow resonance, labeled $\Xi_{b}(6100)^{-}$, is observed at a $\Xi_{b}^{-} \pi^{+} \pi^{-}$invariant mass of $6100.3 \pm 0.2$ (stat) \pm 0.1 (syst) $\pm 0.6\left(\Xi_{b}^{-}\right) \mathrm{MeV}$, where the last uncertainty reflects the precision of the $\Xi_{b}^{-}$baryon mass. The upper limit on the $\Xi_{b}(6100)^{-}$natural width is determined to be $1.9 \mathrm{MeV}$ at $95 \%$ confidence level. The low $\Xi_{b}(6100)^{-}$signal yield observed in data does not allow a measurement of the quantum numbers of the new state. However, following analogies with the established excited $\Xi_{c}$ baryon states, the new $\Xi_{b}(6100)^{-}$resonance and its decay sequence are consistent with the orbitally excited $\Xi_{b}^{-}$baryon, with spin and parity quantum numbers $J^{P}=3 / 2^{-}$.
\end{abstract}

DOI: 10.1103/PhysRevLett.126.252003

The $\Xi_{b}$ baryon family consists of isodoublet states composed of $b s q$ quarks, where $q$ represents an up or a down quark for the $\Xi_{b}^{0}$ and $\Xi_{b}^{-}$states, respectively. According to the quark model for baryons containing one heavy quark [1], three such isodoublets that are neither orbitally nor radially excited should exist, including one with the light diquark angular momentum $j_{q s}=0$ and spin parity $J^{P}=1 / 2^{+}$(the $\Xi_{b}$ ground states), one with $j_{q s}=1$ and $J^{P}=1 / 2^{+}$(the $\Xi_{b}^{\prime}$ ), and one with $j_{q s}=1$ and $J^{P}=$ $3 / 2^{+}$(the $\Xi_{b}^{*}$ ). Various theoretical models and calculations predict a spectrum of excited $\Xi_{b}$ baryons [2-16]. Three of the four excited states with $j_{q s}=1$ have been observed at the CERN LHC [17-19] via their $\Xi_{b}^{-} \pi^{+}$and $\Xi_{b}^{0} \pi^{-}$decays, in agreement with predictions [2-4]. The fourth state, $\Xi_{b}^{\prime 0}$, is expected to be lighter than the $\Xi_{b}^{-} \pi^{+}$mass threshold, making a strong transition to $\Xi_{b}^{-}$kinematically impossible. The next prominent isodoublets, in analogy with the quark model assumptions for the well-established excited $\Xi_{c}$ baryons [20], are orbitally excited $P$-wave $\Xi_{b}^{* *}$ states with $J^{P}=1 / 2^{-}\left(3 / 2^{-}\right)$, expected to decay to $\Xi_{b}^{\prime}\left(\Xi_{b}^{*}\right) \pi$ $[12,13,21]$. Recently, the LHCb Collaboration reported the observation of the $\Xi_{b}(6227)^{-}$[22] and $\Xi_{b}(6227)^{0}$ [23] states, the former decaying to both $\Lambda_{b}^{0} K^{-}$and $\Xi_{b}^{0} \pi^{-}$, and the latter to $\Xi_{b}^{-} \pi^{+}$.

This Letter presents a search for $\Xi_{b}^{-}$excited states in the $\Xi_{b}^{-} \pi^{+} \pi^{-}$invariant mass spectrum, performed using proton-

*Full author list given at the end of the article.

Published by the American Physical Society under the terms of the Creative Commons Attribution 4.0 International license. Further distribution of this work must maintain attribution to the author(s) and the published article's title, journal citation, and DOI. Funded by SCOAP ${ }^{3}$. proton $(p p)$ collision data samples collected by the CMS experiment at the LHC at $\sqrt{s}=13 \mathrm{TeV}$ in 2016-2018, corresponding to an integrated luminosity of $140 \mathrm{fb}^{-1}$. The ground state $\Xi_{b}^{-}$is reconstructed via its decays to $J / \psi \Xi^{-}$ and $J / \psi \Lambda K^{-}$, followed by the decays $J / \psi \rightarrow \mu^{+} \mu^{-}$, $\Xi^{-} \rightarrow \Lambda \pi^{-}$, and $\Lambda \rightarrow p \pi^{-}$. The decay topologies are illustrated in Fig. 1. For the $\Xi_{b}^{-} \rightarrow J / \psi \Lambda K^{-}$decay mode, following the studies reported by the LHCb Collaboration [24], the partially reconstructed $\Xi_{b}^{-} \rightarrow J / \psi \Sigma^{0} K^{-}$channel is also used, where the photon from the $\Sigma^{0} \rightarrow \Lambda \gamma$ decay is too soft to be detected. The inclusion of charge-conjugated states is implied throughout this Letter. A signal peak, hereafter referred to as $\Xi_{b}(6100)^{-}$, is clearly observed near the $\Xi_{b}^{-} \pi^{+} \pi^{-}$kinematic threshold, with a decay sequence consistent with being the $\Xi_{b}(6100)^{-} \rightarrow \Xi_{b}^{* 0} \pi^{-} \rightarrow \Xi_{b}^{-} \pi^{+} \pi^{-}$ decay. The $\Xi_{b}(6100)^{-}$mass and an upper limit on its width are also measured.

The central feature of the CMS apparatus is a superconducting solenoid of $6 \mathrm{~m}$ internal diameter, providing a magnetic field of $3.8 \mathrm{~T}$. Within the solenoid volume are a silicon pixel and strip tracker, a lead tungstate crystal electromagnetic calorimeter, and a brass and scintillator hadron calorimeter, each composed of a barrel and two end cap sections. Muons are detected in gas-ionization chambers embedded in the steel flux-return yoke outside the solenoid. A more detailed description of the CMS detector, together with a definition of the coordinate system used and the relevant kinematic variables, can be found in Ref. [25].

Events of interest are selected using a two-tiered trigger system. The first level (L1), composed of custom hardware processors, uses information from the calorimeters and muon detectors [26]. The second level, known as the highlevel trigger (HLT), consists of a farm of processors 


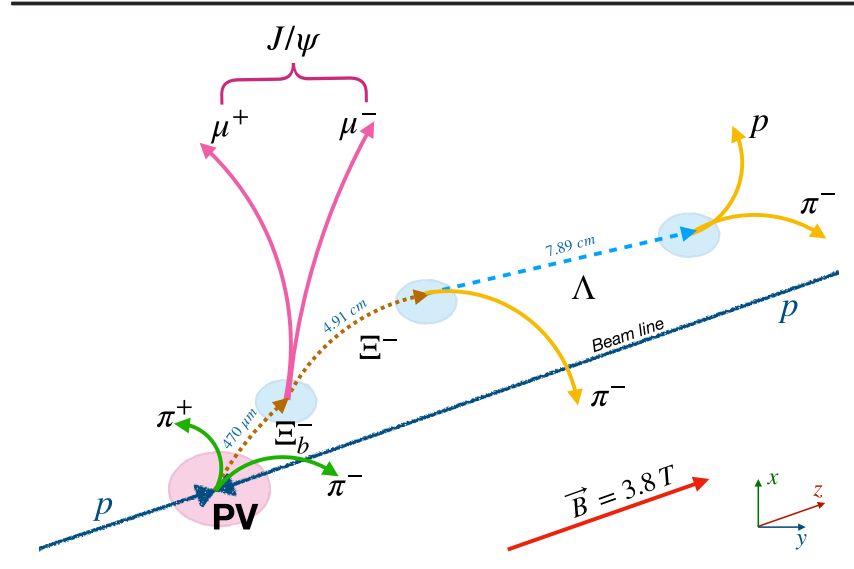

crossings (pileup) with a multiplicity distribution matching the measured one.

The selection criteria are optimized using the Punzi figure of merit [33], which does not rely on the signal normalization. The expected background is estimated from data using the same-sign (SS) control region described below, while the signal efficiency is obtained from the simulated $\Xi_{b}(6100)^{-} \rightarrow \Xi_{b}^{-} \pi^{+} \pi^{-}$events. The $\Xi_{b}^{-} \rightarrow$ $J / \psi \Xi^{-}$and $\Xi_{b}^{-} \rightarrow J / \psi \Lambda K^{-}$requirements are optimized separately.

Events are required to have two OS muons passing the CMS soft-muon selection criteria [34] and satisfying $p_{T}\left(\mu^{ \pm}\right)>3 \mathrm{GeV}$ and $\left|\eta\left(\mu^{ \pm}\right)\right|<2.4$. The muons must form a common vertex with $\chi^{2}$ probability $P_{\mathrm{vtx}}$ above $1 \%$. The dimuon invariant mass must be within $100 \mathrm{MeV}$ of $m_{J / \psi}^{\mathrm{PDG}}$ (hereafter, $m_{X}^{\mathrm{PDG}}$ denotes the world-average mass of hadron $X[20])$, corresponding to about three times the mass resolution. The $\Lambda$ candidates are formed from displaced two-prong vertices, assuming the decay $\Lambda \rightarrow p \pi^{-}$, as described in Ref. [35]. The $p \pi^{-}$reconstructed mass is required to be within $10 \mathrm{MeV}$ of $m_{\Lambda}^{\mathrm{PDG}}$, corresponding to about three times the mass resolution. The two tracks are then refitted with their invariant mass constrained to $m_{\Lambda}^{\text {PDG }}$. The obtained $\Lambda$ candidates are required to have $p_{T}>1 \mathrm{GeV}$ and $P_{\mathrm{vtx}}>1 \%$.

For the $\Xi_{b}^{-} \rightarrow J / \psi \Xi^{-}$channel, the $\Xi^{-} \rightarrow \Lambda \pi^{-}$candidates are obtained by combining charged particles of $p_{T}>$ $0.25 \mathrm{GeV}$ with the selected $\Lambda$ candidates. The reconstructed $\Xi^{-}$must have $P_{\mathrm{vtx}}>1 \%, p_{T}>3 \mathrm{GeV}$, and invariant mass within $9.5 \mathrm{MeV}$ of $m_{\Xi^{-}}^{\mathrm{PDG}}$, corresponding to about three times the mass resolution. The $\Xi_{b}^{-}$candidates are obtained by performing a $\mu^{+} \mu^{-} \Xi^{-}$kinematic vertex fit, constraining the dimuon invariant mass to $m_{J / \psi}^{\mathrm{PDG}}$.

For the $\Xi_{b}^{-} \rightarrow J / \psi \Lambda K^{-}$decay channel, the $\Lambda$ candidates must pass stricter requirements: $p_{T}>2 \mathrm{GeV}$ and $\left|M\left(p \pi^{-}\right)-m_{\Lambda}^{\mathrm{PDG}}\right|<9 \mathrm{MeV}$. The charged kaon candidates are particle tracks with kaon mass assignment satisfying high-purity tracking requirements [36] and $p_{T}>1.2 \mathrm{GeV}$. The $\Xi_{b}^{-}$candidates are reconstructed by fitting the $\mu^{+} \mu^{-} \Lambda K^{-}$vertex with the $J / \psi$ mass constraint. Because the photon from the $\Sigma^{0} \rightarrow \Lambda \gamma$ decay is not detected, both $\Xi_{b}^{-} \rightarrow J / \psi \Lambda K^{-}$and $\Xi_{b}^{-} \rightarrow J / \psi \Sigma^{0} K^{-}$decays contribute to the $\mu^{+} \mu^{-} \Lambda K^{-}$reconstructed combination.

The $\Xi_{b}^{-}$candidates are required to have $P_{\mathrm{vtx}}>1 \%$ and $p_{T}>10(15) \mathrm{GeV}$ for the $\Xi_{b}^{-} \rightarrow J / \psi \Xi^{-}\left(\Xi_{b}^{-} \rightarrow J / \psi \Lambda K^{-}\right)$ channel. From all reconstructed $p p$ collision vertices, the primary vertex (PV) is chosen as the one with the smallest pointing angle, as done in Refs. [37-40]. The pointing angle is the three-dimensional angle between the $\Xi_{b}^{-}$ candidate momentum and the vector joining the PV with the reconstructed $\Xi_{b}^{-}$candidate decay vertex. The decay length $L_{x y}$ of the $\Xi_{b}^{-}$candidate in the transverse plane, computed as the two-dimensional distance between the PV and the $\Xi_{b}^{-}$decay vertex, is required to be at least three times larger than its uncertainty $\sigma_{L_{x y}}$. The $\overrightarrow{p_{T}}\left(\Xi_{b}^{-}\right)$is 
required to be aligned with the transverse displacement vector: $\cos \left[\alpha\left(\Xi_{b}^{-}, \mathrm{PV}\right)\right]>0.99(0.993)$ for the $\Xi_{b}^{-} \rightarrow$ $J / \psi \Xi^{-}\left(\Xi_{b}^{-} \rightarrow J / \psi \Lambda K^{-}\right)$channel, where $\alpha\left(\Xi_{b}^{-}, \mathrm{PV}\right)$ is the pointing angle in the plane transverse to the beams. Two additional topological requirements are applied: the cosine of the pointing angle $\cos \left[\alpha\left(\Xi^{-}, \Xi_{b}^{-}\right)\right]$must be larger than 0.999 for the $\Xi_{b}^{-} \rightarrow J / \psi \Xi^{-}$channel; and $L_{x y} / \sigma_{L_{x y}}\left(\Lambda, \Xi_{b}^{-}\right)>20$ for the $\Xi_{b}^{-} \rightarrow J / \psi \Lambda K^{-}$channel. In addition, the pion emitted in the $\Xi^{-} \rightarrow \Lambda \pi^{-}$decay and the kaon emitted in the $\Xi_{b}^{-} \rightarrow J / \psi \Lambda K^{-}$decay must have $d_{x y} / \sigma_{d_{x y}}>0.9$ and 0.6 , respectively, where $d_{x y}$ is the impact parameter in the transverse plane with respect to the $\mathrm{PV}$, and $\sigma_{d_{x y}}$ is its uncertainty.

The invariant mass distributions of the selected $\Xi_{b}^{-}$ candidates are shown in Fig. 2 for the $J / \psi \Xi^{-}$(upper) and $J / \psi \Lambda K^{-}$(lower) channels. The two plots also show the results of independent unbinned extended maximum-likelihood fits. In both cases, the fully reconstructed $\Xi_{b}^{-}$signal is described by a double-Gaussian function with two free
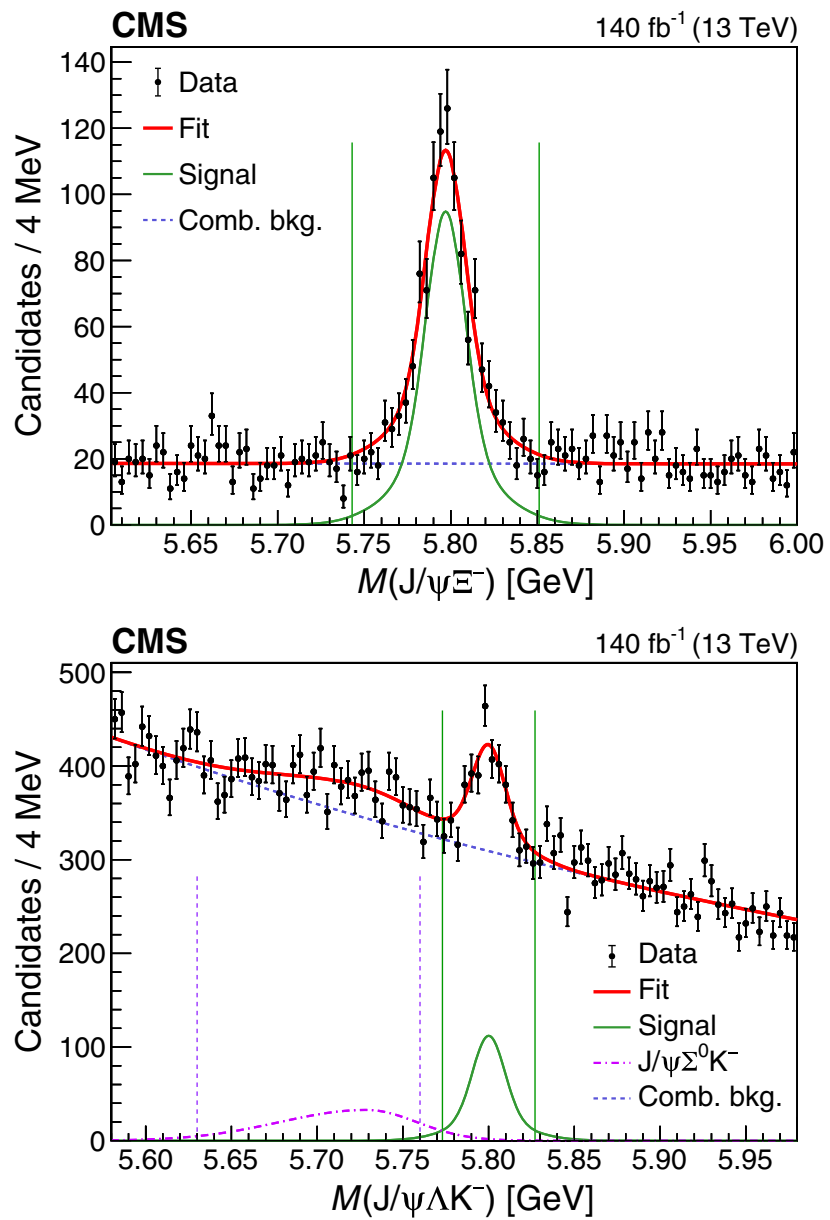

FIG. 2. Invariant mass distributions of the selected $\Xi_{b}^{-}$candidates in the $J / \psi \Xi^{-}$(upper) and $J / \psi \Lambda K^{-}$(lower) decay channels with the fit results superimposed. The vertical solid (dashed) lines show the mass windows discussed in the text and used in the reconstruction of the $\Xi_{b}^{-} \pi^{+} \pi^{-}$candidates in $J / \psi \Xi^{-}$and $J / \psi \Lambda K^{-}$ $\left(J / \psi \Sigma^{0} K^{-}\right)$channels. parameters: the common mean and the total yield; the two width parameters and the proportion of each Gaussian are fixed from simulation studies. The background is described by a first-order polynomial in the $J / \psi \Xi^{-}$fit and an exponential function in the $J / \psi \Lambda K^{-}$fit. In the latter fit, the signal contribution from the partially reconstructed $\Xi_{b}^{-} \rightarrow J / \psi \Sigma^{0} K^{-}$decays is taken into account by including an asymmetric Gaussian in the fit model, with the shape parameters fixed from simulation studies. All normalization values (signals and backgrounds) are free parameters of the fit.

The signal yields from the fits described above are $859 \pm 36$ and $815 \pm 74$ for the $\Xi_{b}^{-} \rightarrow J / \psi \Xi^{-}$and fully reconstructed $\Xi_{b}^{-} \rightarrow J / \psi \Lambda K^{-}$decay modes, respectively, with the uncertainties being statistical only. The fitted $\Xi_{b}^{-}$ masses of $5797.0 \pm 0.7$ and $5800.1 \pm 1.2 \mathrm{MeV}$, respectively for the $J / \psi \Xi^{-}$and $J / \psi \Lambda K^{-}$channels, the uncertainties being statistical only, are consistent with each other and with the world-average value, $5797.0 \pm 0.6 \mathrm{MeV}$ [20]. The signal components corresponding to fully reconstructed $\Xi_{b}^{-}$candidates are shown by the solid green curves. The fitted yield of the partially reconstructed $\Xi_{b}^{-} \rightarrow J / \psi \Sigma^{0} K^{-}$contribution, reconstructed as $J / \psi \Lambda K^{-}$, is $820 \pm 158$, represented by the dotted-dashed curve in Fig. 2 (lower). The $\Xi_{b}^{-}$fit results illustrate this part of the reconstruction procedure and provide the first confirmation of the $\Xi_{b}^{-} \rightarrow J / \psi \Lambda K^{-}$decay observed by LHCb [24].

When reconstructing $\Xi_{b}^{-} \pi^{+} \pi^{-}$candidates, we select events with $\Xi_{b}^{-}$invariant mass within 54 (27) $\mathrm{MeV}$ of the fitted $\Xi_{b}^{-}$mass for the $J / \psi \Xi^{-}\left(J / \psi \Lambda K^{-}\right)$channel, corresponding to approximately 2.8 (1.8) times the mass resolution, as shown by the vertical solid lines in Fig. 2. The $5.63<M\left(J / \psi \Lambda K^{-}\right)<5.76 \mathrm{GeV}$ mass region is used for the partially reconstructed $\Xi_{b}^{-} \rightarrow J / \psi \Sigma^{0} K^{-}$decay mode, shown by the dashed vertical lines in Fig. 2 (lower). These mass ranges are selected through the same optimization procedure as used for the other selection criteria.

Because the lifetime of the excited $\Xi_{b}$ states is expected to be negligible, the $\Xi_{b}^{-} \pi^{+} \pi^{-}$candidates are formed by combining the selected $\Xi_{b}^{-}$candidates with two OS tracks originating from the PV, as in Refs. [37-40]. Combinations of a $\Xi_{b}^{-}$candidate with two SS pions from the PV are used as a control channel and form the SS control region. The analysis is performed using the mass difference variable $\Delta M=M\left(\Xi_{b}^{-} \pi^{+} \pi^{-}\right)-M\left(\Xi_{b}^{-}\right)-2 m_{\pi^{ \pm}}^{\mathrm{PDG}}$, which has a better mass resolution than $M\left(\Xi_{b}^{-} \pi^{+} \pi^{-}\right)$, where $M\left(\Xi_{b}^{-}\right)$represents the reconstructed $\Xi_{b}^{-}$mass. According to the simulation studies, this variable also has the advantage of being insensitive to a potential mass shift caused by the fact that the photon emitted in the $\Xi_{b}^{-} \rightarrow J / \psi \Sigma^{0} K^{-}, \Sigma^{0} \rightarrow \Lambda \gamma$ decay sequence is not reconstructed. Following the technique developed in Ref. [40], the selected $\Xi_{b}^{-}$candidate and all tracks forming the PV are refit to a common vertex, further improving the $\Xi_{b}^{-} \pi^{+} \pi^{-}$invariant mass resolution of the fully reconstructed channels from $1.39 \pm 0.11$ to 
$0.94 \pm 0.06 \mathrm{MeV}$ (statistical uncertainties only), as obtained from simulation studies.

Theoretical studies [12,13,21] and analogous decays of excited charm baryons [20,41] suggest that the decay $\Xi_{b}^{* *-} \rightarrow \Xi_{b}^{-} \pi^{+} \pi^{-}$should proceed predominantly through $\Xi_{b}^{* *-} \rightarrow \Xi_{b}^{* 0} \pi^{-}$, followed by $\Xi_{b}^{* 0} \rightarrow \Xi_{b}^{-} \pi^{+}$. Therefore, an additional requirement is applied to enhance this contribution. As the $\Xi_{b}^{* 0}$ state has a mass of $5952.3 \pm 0.6 \mathrm{MeV}$, the mass difference $M\left(\Xi_{b}^{* 0}\right)-M\left(\Xi_{b}^{-}\right)-m_{\pi^{+}}^{\mathrm{PDG}}$ will peak at 15.73 MeV [20]. To avoid complications in understanding the $\Xi_{b}^{-} \pi^{+} \pi^{-}$threshold, we do not apply a minimum cut on this mass difference but simply require it to be less than $20.73 \mathrm{MeV}$, with the $5 \mathrm{MeV}$ addition found to be optimal when considering the $\Xi_{b}^{* 0}$ natural width and our detector resolution.

The invariant mass distribution of the selected $\Xi_{b}^{-} \pi^{+} \pi^{-}$ candidates is shown in Fig. 3, using the mass difference variable $\Delta M$. The left plot combines the data from the $\Xi_{b}^{-} \rightarrow J / \psi \Xi^{-}$and $\Xi_{b}^{-} \rightarrow J / \psi \Lambda K^{-}$channels, which have
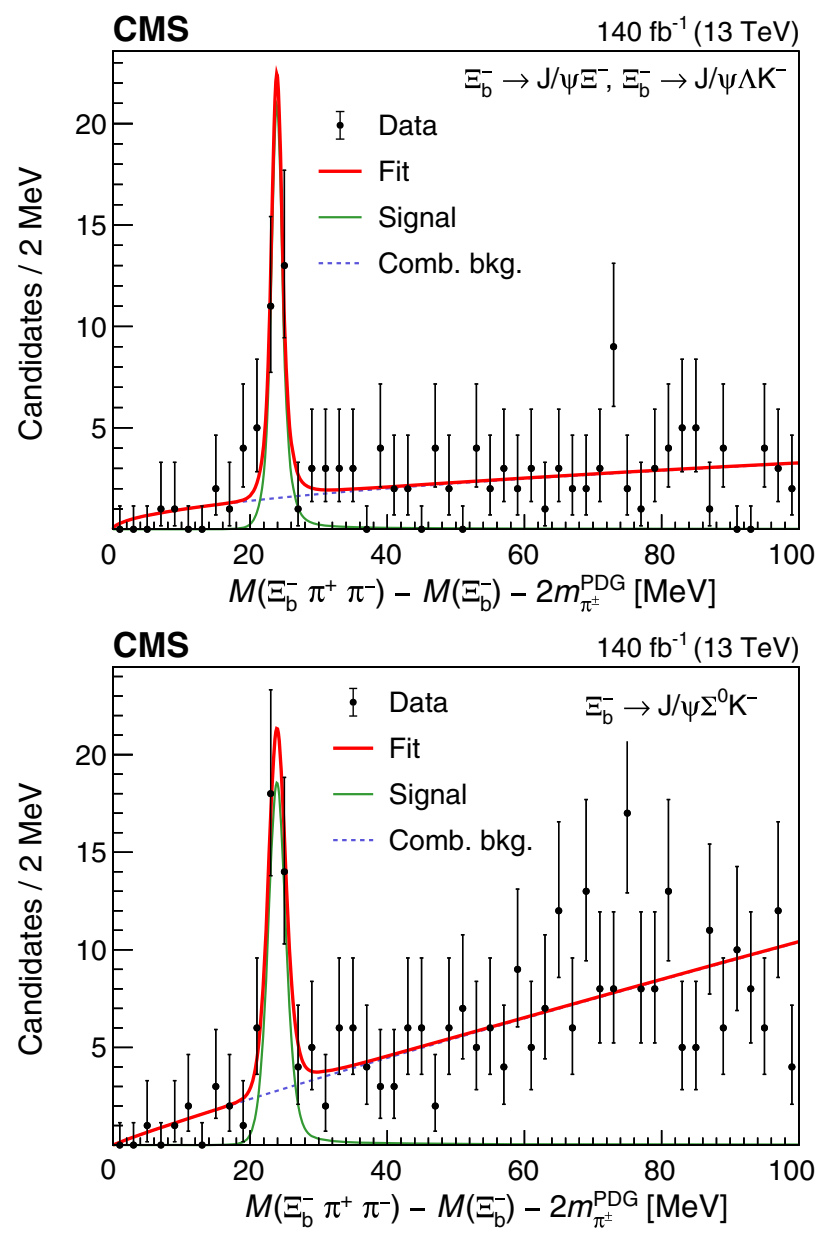

FIG. 3. Distributions of the invariant mass difference $\Delta M$ for the selected $\Xi_{b}^{-} \pi^{+} \pi^{-}$candidates, with the $\Xi_{b}^{-}$reconstructed in the $J / \psi \Xi^{-}$and $J / \psi \Lambda K^{-}$channels (upper) or partially reconstructed in the $J / \psi \Sigma^{0} K^{-}$channel (lower). The result of the simultaneous fit is also shown. identical mass resolutions, according to simulation studies (the $\Xi_{b}^{-}$is fully reconstructed in both channels). The right plot shows the events that use the partially reconstructed $\Xi_{b}^{-} \rightarrow J / \psi \Sigma^{0} K^{-}$channel, with a $30 \%$ larger mass resolution. Given the definition of the $\Delta M$ variable, the mean mass of the signal peaks should not depend on the $\Xi_{b}^{-}$ reconstruction channel.

A narrow peak is seen near the threshold of the $\Xi_{b}^{-} \pi^{+} \pi^{-}$ system in both plots of Fig. 3. The excess is also visible in each of the two independent decay channels, $J / \psi \Xi^{-}$and $J / \psi \Lambda K^{-}$. We have also studied the OS and SS distributions in a wider range of $\Delta M$ (up to $280 \mathrm{MeV}$ ) and found no other significant peaks. A simultaneous unbinned extended maximum-likelihood fit is performed on the two data samples shown in Fig. 3, the result being represented by the red curves. The signal component is described with a relativistic Breit-Wigner (RBW) function $[42,43]$ for the $\Xi_{b}(6100)^{-} \rightarrow$ $\Xi_{b}^{* 0} \pi^{-}$decay, convolved with a double-Gaussian resolution function. The mass and natural width of the signal function are the two parameters of interest in the fit. The normalization and background parameters are different for the fully and partially reconstructed channels, as are the resolution parameters, which are fixed from the simulation studies. The background component is modeled with the threshold function $(\Delta M)^{\alpha}$, where $\alpha$ is a free parameter.

The fitted mass difference of the new $\Xi_{b}(6100)^{-}$state is $\Delta M_{\Xi_{b}(6100)^{-}}=24.14 \pm 0.22 \mathrm{MeV}$, where the uncertainty is statistical only. The fitted signal yields are $26 \pm 7$ and $34 \pm 9$ for the fully reconstructed and the $\Xi_{b}^{-} \rightarrow J / \psi \Sigma^{0} K^{-}$ channels, respectively. The relative yield of the $\Xi_{b}(6100)^{-}$, with respect to the $\Xi_{b}^{-}$yield, is found to be noticeably larger in the partially reconstructed $\Xi_{b}^{-}$channel, compared to the fully reconstructed channels. Given the large uncertainties in the observed small $\Xi_{b}(6100)^{-}$signal yields, this discrepancy is consistent with being a statistical fluctuation. The order-of-magnitude larger signals of the $\Xi_{b}^{* 0} \rightarrow \Xi_{b}^{-} \pi^{+}$ decays, with respect to the $\Xi_{b}^{-}$ground state signals, are found to be consistent between all three $\Xi_{b}^{-}$reconstruction channels.

The natural width of the $\Xi_{b}(6100)^{-}$is too small to be measured with the present data sample and experimental resolution. An upper limit on $\Gamma\left[\Xi_{b}(6100)^{-}\right]$has been obtained through a scan of the profiled likelihood, assuming an asymptotic distribution. The measured upper limit, at 95\% confidence level, is $\Gamma\left[\Xi_{b}(6100)^{-}\right]<1.9 \mathrm{MeV}$, where the systematic uncertainties, discussed below, are taken into account.

The local statistical significance of the $\Xi_{b}(6100)^{-}$signal is evaluated with the likelihood ratio technique, comparing the background-only and signal-plus-background hypotheses (with four additional free parameters), using asymptotic formulas $[44,45]$. The resulting significance of the $\Xi_{b}(6100)^{-}$signal varies between 6.2 and 6.7 standard deviations, depending on the fit model variations used to evaluate the systematic uncertainties. 
Several sources of systematic uncertainties in the measured mass difference $\Delta M_{\Xi_{b}(6100)^{-}}$are considered. To evaluate the systematic uncertainties related to the choice of the fit model, several alternative functions are tested. Uncertainties related to the choice of the signal model are estimated by changing the resolution function from a double-Gaussian function to a single-Gaussian function or a sum of three Gaussian functions. Two alternative background models are considered: the threshold function multiplied by an exponential and the threshold function multiplied by a first-order polynomial. The largest deviations in the measured mass are 0.01 and $0.04 \mathrm{MeV}$, respectively, for the variations of the signal and background models; these values are taken as the two corresponding systematic uncertainties.

The RBW function used in the signal modeling includes Blatt-Weisskopf barrier factors [43], which depend on the radial parameter $r$ and on the angular momentum $l$ (spin). In the baseline fit, $r=3.5 \mathrm{GeV}^{-1}$ and $l=1$. The corresponding systematic uncertainties are obtained by varying $r$ between 1 and $5 \mathrm{GeV}^{-1}$ or by assigning $l=0$. The $r$ variations have a negligible effect on the results, while fixing $l=0$ changes the signal shape and induces a mass difference variation of $0.01 \mathrm{MeV}$, taken as the corresponding systematic uncertainty.

To account for a possible difference between the measured and simulated mass resolutions, the fits are repeated with resolutions scaled up or down by 1.074 , a factor determined from the comparison of the $\Xi_{b}^{-}$resolutions in data and simulation. The resulting systematic uncertainty of the $\Xi_{b}(6100)^{-}$mass difference is $0.02 \mathrm{MeV}$.

Simulation studies show a shift of $0.07 \mathrm{MeV}$ between the generated and reconstructed mass differences; this shift is treated as an additional systematic uncertainty in the $\Delta M_{\Xi_{b}(6100)^{-}}$measurement.

The systematic uncertainty reflecting the $\Delta M$ fit range is evaluated by changing the upper end of the $\Delta M$ fit range from its default $100 \mathrm{MeV}$ to 80,120 , and $150 \mathrm{MeV}$. The largest mass difference change of $0.02 \mathrm{MeV}$ is taken as the corresponding systematic uncertainty.

A potential bias due to a possible misalignment of the tracker detectors is evaluated by comparing the results obtained with the data collected in 2016, 2017, and 2018. This is a reasonable evaluation, given that the inner part of the CMS tracker was replaced between the 2016 and 2017 data-taking periods. The measured mass is found to be insensitive to alignment uncertainties.

The total systematic uncertainty in the measured mass difference $\Delta M_{\Xi_{b}(6100)^{-}}$, calculated as the sum in quadrature of the partial terms, is $0.09 \mathrm{MeV}$.

In summary, we report the observation of a new excited beauty strange baryon, decaying to $\Xi_{b}^{-} \pi^{+} \pi^{-}$. The analysis uses proton-proton collision data collected by the CMS experiment at $\sqrt{s}=13 \mathrm{TeV}$, corresponding to an integrated luminosity of $140 \mathrm{fb}^{-1}$. The measured mass difference of this state is $M\left[\Xi_{b}(6100)^{-}\right]-$ $M\left(\Xi_{b}^{-}\right)-2 m_{\pi^{ \pm}}^{\mathrm{PDG}}=24.14 \pm 0.22$ (stat) \pm 0.09 (syst) $\mathrm{MeV}$. The known $\Xi_{b}^{-}$mass of $5797.0 \pm 0.6 \mathrm{MeV}$ [20] is used to obtain $M\left[\Xi_{b}(6100)^{-}\right]=6100.3 \pm 0.2($ stat $) \pm$ 0.1 (syst) $\pm 0.6\left(\Xi_{b}^{-}\right) \mathrm{MeV}$. It is particularly remarkable that if the $\Xi_{b}(6100)^{-}$baryon were only $13 \mathrm{MeV}$ heavier, it would be above the $\Lambda_{b}^{0} K^{-}$mass threshold and could decay to this final state. The natural width of this resonance is compatible with zero and a 95\% confidence level upper limit of $1.9 \mathrm{MeV}$ has been determined.

Following analogies with the established excited $\Xi_{c}$ baryon states [20], and considering several theoretical predictions $[12,13,21]$, the new $\Xi_{b}(6100)^{-}$resonance and its decay sequence are consistent with the lightest orbitally excited $\Xi_{b}^{-}$baryon, with the light diquark angular momentum $j_{d s}=1$ and $J^{P}=3 / 2^{-}$(excitation with orbital momentum $L=1$ between the b quark and the $d s$ diquark). This suggests that it is the beauty analog of the $\Xi_{c}(2815)$ baryon [41]. Measuring a natural width of the $\Xi_{b}(6100)^{-}$ smaller than $1.9 \mathrm{MeV}$ comes as a surprise, given the larger values predicted by the theory calculations $[12,13,21]$, based on the assumption that the $\Xi_{b}^{* *-} \rightarrow \Xi_{b}^{* 0} \pi^{-}$decay proceeds predominantly via $S$ wave $\left(3 / 2^{-} \rightarrow 3 / 2^{+} 0^{-}\right)$. The observation of this baryon and the measurement of its properties provide information that should help to distinguish between different theoretical models used to calculate the properties of the excited $\Xi_{b}$ states.

We congratulate our colleagues in the CERN accelerator departments for the excellent performance of the LHC and thank the technical and administrative staffs at CERN and at other CMS institutes for their contributions to the success of the CMS effort. In addition, we gratefully acknowledge the computing centers and personnel of the Worldwide LHC Computing Grid and other centers for delivering so effectively the computing infrastructure essential to our analyses. Finally, we acknowledge the enduring support for the construction and operation of the LHC, the CMS detector, and the supporting computing infrastructure provided by the following funding agencies: BMBWF and FWF (Austria); FNRS and FWO (Belgium); CNPq, CAPES, FAPERJ, FAPERGS, and FAPESP (Brazil); MES (Bulgaria); CERN; CAS, MoST, and NSFC (China); COLCIENCIAS (Colombia); MSES and CSF (Croatia); RIF (Cyprus); SENESCYT (Ecuador); MoER, ERC PUT, and ERDF (Estonia); Academy of Finland, MEC, and HIP (Finland); CEA and CNRSIN2P3 (France); BMBF, DFG, and HGF (Germany); GSRT (Greece); NKFIA (Hungary); DAE and DST (India); IPM (Iran); SFI (Ireland); INFN (Italy); MSIP and NRF (Republic of Korea); MES (Latvia); LAS (Lithuania); MOE and UM (Malaysia); BUAP, CINVESTAV, CONACYT, LNS, SEP, and UASLP-FAI 
(Mexico); MOS (Montenegro); MBIE (New Zealand); PAEC (Pakistan); MSHE and NSC (Poland); FCT (Portugal); JINR (Dubna); MON, RosAtom, RAS, RFBR, and NRC KI (Russia); MESTD (Serbia); SEIDI, CPAN, PCTI, and FEDER (Spain); MOSTR (Sri Lanka); Swiss Funding Agencies (Switzerland); MST (Taipei); ThEPCenter, IPST, STAR, and NSTDA (Thailand); TUBITAK and TAEK (Turkey); NASU (Ukraine); STFC (United Kingdom); and DOE and NSF (USA). Individuals have received support from the Marie Curie program and the European Research Council and Horizon 2020 Grant, Contracts No. 675440, No. 724704, No. 752730, and No. 765710 (European Union); the Leventis Foundation; the Alfred P. Sloan Foundation; the Alexander von Humboldt Foundation; the Belgian Federal Science Policy Office; the Fonds pour la Formation à la Recherche dans l'Industrie et dans l'Agriculture (FRIA-Belgium); the Agentschap voor Innovatie door Wetenschap en Technologie (IWTBelgium); the F. R. S.-FNRS and FWO (Belgium) under the "Excellence of Science-EOS"-be.h Project No. 30820817; the Beijing Municipal Science \& Technology Commission, No. Z191100007219010; the Ministry of Education, Youth and Sports (MEYS) of the Czech Republic; the Deutsche Forschungsgemeinschaft (DFG), under Germany's Excellence Strategy-EXC 2121 "Quantum Universe"-390833306, and under Project No. 400140256-GRK2497; the Lendület ("Momentum") Program and the János Bolyai Research Scholarship of the Hungarian Academy of Sciences, the New National Excellence Program ÚNKP, the NKFIA Research Grants No. 123842, No. 123959, No. 124845, No. 124850 , No. 125105 , No. 128713 , No. 128786 , and No. 129058 (Hungary); the Council of Science and Industrial Research, India; the HOMING PLUS program of the Foundation for Polish Science, cofinanced from European Union, Regional Development Fund, the Mobility Plus program of the Ministry of Science and Higher Education, the National Science Center (Poland), Contracts Harmonia 2014/14/M/ST2/00428, Opus 2014/ 13/B/ST2/02543, 2014/15/B/ST2/03998, and 2015/19/B/ ST2/02861, Sonata-bis 2012/07/E/ST2/01406; the National Priorities Research Program by Qatar National Research Fund; the Ministry of Science and Higher Education, Project No. 14.W03.31.0026 (Russia); the Programa Estatal de Fomento de la Investigación Científica y Técnica de Excelencia María de Maeztu, Grant No. MDM-2015-0509 and the Programa Severo Ochoa del Principado de Asturias; the Thalis and Aristeia programs cofinanced by EU-ESF and the Greek NSRF; the Rachadapisek Sompot Fund for Postdoctoral Fellowship, Chulalongkorn University and the Chulalongkorn Academic into Its 2nd Century Project Advancement Project (Thailand); the Kavli Foundation; the Nvidia Corporation; the SuperMicro Corporation; the
Welch Foundation, Contract No. C-1845; and the Weston Havens Foundation (USA).

[1] D. Ebert, T. Feldmann, C. Kettner, and H. Reinhardt, A diquark model for baryons containing one heavy quark, $\mathrm{Z}$. Phys. C 71, 329 (1996).

[2] D. Ebert, R. N. Faustov, and V. O. Galkin, Masses of excited heavy baryons in the relativistic quark model, Phys. Lett. B 659, 612 (2008).

[3] E. E. Jenkins, Model-independent bottom baryon mass predictions in the $1 / \mathrm{N}(c)$ expansion, Phys. Rev. D 77, 034012 (2008).

[4] M. Karliner, B. Keren-Zur, H. J. Lipkin, and J. L. Rosner, The quark model and b baryons, Ann. Phys. (Amsterdam) 324, 2 (2009).

[5] W. Roberts and M. Pervin, Heavy baryons in a quark model, Int. J. Mod. Phys. A 23, 2817 (2008).

[6] D. Ebert, R. N. Faustov, and V. O. Galkin, Spectroscopy and Regge trajectories of heavy baryons in the relativistic quarkdiquark picture, Phys. Rev. D 84, 014025 (2011).

[7] H. Garcilazo, J. Vijande, and A. Valcarce, Faddeev study of heavy baryon spectroscopy, J. Phys. G 34, 961 (2007).

[8] B. Chen, K.-W. Wei, and A. Zhang, Assignments of $\Lambda_{Q}$ and $\Xi_{Q}$ baryons in the heavy quark-light diquark picture, Eur. Phys. J. A 51, 82 (2015).

[9] I. L. Grach, I. M. Narodetskii, M. A. Trusov, and A. I. Veselov, Heavy baryon spectroscopy in the QCD string model, in Proceedings of the 18th International Conference on Particles and Nuclei (PANIC08) (2008) [arXiv:0811.2184].

[10] Q. Mao, H.-X. Chen, W. Chen, A. Hosaka, X. Liu, and S.-L. Zhu, QCD sum rule calculation for P-wave bottom baryons, Phys. Rev. D 92, 114007 (2015).

[11] Z.-G. Wang, Analysis of the $1 / 2^{-}$and $3 / 2^{-}$heavy and doubly heavy baryon states with QCD sum rules, Eur. Phys. J. A 47, 81 (2011).

[12] K.-L. Wang, Y.-X. Yao, X.-H. Zhong, and Q. Zhao, Strong and radiative decays of the low-lying $S$ - and $P$-wave singly heavy baryons, Phys. Rev. D 96, 116016 (2017).

[13] Y. Kawakami and M. Harada, Singly heavy baryons with chiral partner structure in a three-flavor chiral model, Phys. Rev. D 99, 094016 (2019).

[14] Z.-Y. Wang, J.-J. Qi, X.-H. Guo, and K.-W. Wei, Spectra of charmed and bottom baryons with hyperfine interaction, Chin. Phys. C 41, 093103 (2017).

[15] K. Thakkar, Z. Shah, A. K. Rai, and P. C. Vinodkumar, Excited state mass spectra and Regge trajectories of bottom baryons, Nucl. Phys. A965, 57 (2017).

[16] K.-W. Wei, B. Chen, N. Liu, Q.-Q. Wang, and X.-H. Guo, Spectroscopy of singly, doubly, and triply bottom baryons, Phys. Rev. D 95, 116005 (2017).

[17] CMS Collaboration, Observation of a New $\Xi_{b}$ Baryon, Phys. Rev. Lett. 108, 252002 (2012).

[18] LHCb Collaboration, Measurement of the properties of the $\Xi_{b}^{* 0}$ baryon, J. High Energy Phys. 05 (2016) 161.

[19] LHCb Collaboration, Observation of Two New $\Xi_{b}^{-}$Baryon Resonances, Phys. Rev. Lett. 114, 062004 (2015). 
[20] Particle Data Group, P. A. Zyla et al., Review of particle physics, Prog. Theor. Exp. Phys. 2020, 083C01 (2020).

[21] B. Chen, K.-W. Wei, X. Liu, and A. Zhang, Role of newly discovered $\Xi_{b}(6227)^{-}$for constructing excited bottom baryon family, Phys. Rev. D 98, 031502(R) (2018).

[22] LHCb Collaboration, Observation of a New $\Xi_{b}^{-}$Resonance, Phys. Rev. Lett. 121, 072002 (2018).

[23] LHCb Collaboration, Observation of a new $\Xi_{b}^{0}$ state, Phys. Rev. D 103, 012004 (2021).

[24] LHCb Collaboration, Observation of the $\Xi_{b}^{-} \rightarrow J / \psi \Lambda K^{-}$ decay, Phys. Lett. B 772, 265 (2017).

[25] CMS Collaboration, The CMS experiment at the CERN LHC, J. Instrum. 3, S08004 (2008).

[26] CMS Collaboration, Performance of the CMS Level-1 trigger in proton-proton collisions at $\sqrt{s}=13 \mathrm{TeV}$, J. Instrum. 15, P10017 (2020).

[27] CMS Collaboration, The CMS trigger system, J. Instrum. 12, P01020 (2017).

[28] T. Sjöstrand, S. Ask, J. R. Christiansen, R. Corke, N. Desai, P. Ilten, S. Mrenna, S. Prestel, C. O. Rasmussen, and P.Z. Skands, An introduction to PYTHIA 8.2, Comput. Phys. Commun. 191, 159 (2015).

[29] D. J. Lange, The EVTGEN particle decay simulation package, Nucl. Instrum. Methods Phys. Res., Sect. A 462, 152 (2001).

[30] E. Barberio, B. van Eijk, and Z. Wąs, Рнотоs: A universal Monte Carlo for QED radiative corrections in decays, Comput. Phys. Commun. 66, 115 (1991).

[31] E. Barberio and Z. Wąs, Pнотоs: A universal Monte Carlo for QED radiative corrections. Version 2.0, Comput. Phys. Commun. 79, 291 (1994).

[32] S. Agostinelli et al. (GEANT4 Collaboration), GEANT4-a simulation toolkit, Nucl. Instrum. Methods Phys. Res., Sect. A 506, 250 (2003).

[33] G. Punzi, Sensitivity of searches for new signals and its optimization, eConf C030908, MODT002 (2003) [arXiv: physics/0308063].
[34] CMS Collaboration, Performance of the CMS muon detector and muon reconstruction with proton-proton collisions at $\sqrt{s}=13 \mathrm{TeV}$, J. Instrum. 13, P06015 (2018).

[35] CMS Collaboration, CMS tracking performance results from early LHC operation, Eur. Phys. J. C 70, 1165 (2010).

[36] CMS Collaboration, Description and performance of track and primary-vertex reconstruction with the CMS tracker, J. Instrum. 9, P10009 (2014).

[37] CMS Collaboration, Search for the X(5568) State Decaying Into $\mathrm{B}_{\mathrm{s}}^{0} \pi^{ \pm}$in Proton-Proton Collisions at $\sqrt{s}=8 \mathrm{TeV}$, Phys. Rev. Lett. 120, 202005 (2018).

[38] CMS Collaboration, Studies of $\mathrm{B}_{\mathrm{s} 2}^{*}(5840)^{0}$ and $\mathrm{B}_{\mathrm{s} 1}(5830)^{0}$ mesons including the observation of the $\mathrm{B}_{\mathrm{s} 2}^{*}(5840)^{0} \rightarrow$ $\mathrm{B}^{0} \mathrm{~K}_{\mathrm{S}}^{0}$ decay in proton-proton collisions at $\sqrt{s}=8 \mathrm{TeV}$, Eur. Phys. J. C 78, 939 (2018).

[39] CMS Collaboration, Observation of Two Excited $B_{c}^{+}$States and Measurement of the $\mathrm{B}_{\mathrm{c}}^{+}(2 \mathrm{~S})$ Mass in $p p$ Collisions at $\sqrt{s}=13 \mathrm{TeV}$, Phys. Rev. Lett. 122, 132001 (2019).

[40] CMS Collaboration, Study of excited $\Lambda_{\mathrm{b}}^{0}$ states decaying to $\Lambda_{\mathrm{b}}^{0} \pi^{+} \pi^{-}$in proton-proton collisions at $\sqrt{s}=13 \mathrm{TeV}$, Phys. Lett. B 803, 135345 (2020).

[41] J. P. Alexander et al. (CLEO Collaboration), Evidence of New States Decaying Into $\Xi_{c}^{*} \pi$, Phys. Rev. Lett. 83, 3390 (1999).

[42] J. D. Jackson, Remarks on the phenomenological analysis of resonances, Nuovo Cimento 34, 1644 (1964).

[43] J. M. Blatt and V. F. Weisskopf, Theoretical Nuclear Physics (Springer, New York, 1952), https://doi.org/10.1007/ 978-1-4612-9959-2.

[44] S. S. Wilks, The large-sample distribution of the likelihood ratio for testing composite hypotheses, Ann. Math. Stat. 9, 60 (1938).

[45] G. Cowan, K. Cranmer, E. Gross, and O. Vitells, Asymptotic formulae for likelihood-based tests of new physics, Eur. Phys. J. C 71, 1554 (2011); Erratum, Eur. Phys. J. C 73, 2501 (2013).

A. M. Sirunyan, ${ }^{1, a}$ A. Tumasyan, ${ }^{1}$ W. Adam, ${ }^{2}$ J. W. Andrejkovic, ${ }^{2}$ T. Bergauer, ${ }^{2}$ S. Chatterjee, ${ }^{2}$ M. Dragicevic, ${ }^{2}$ A. Escalante Del Valle, ${ }^{2}$ R. Frühwirth, ${ }^{2, b}$ M. Jeitler, ${ }^{2, b}$ N. Krammer, ${ }^{2}$ L. Lechner, ${ }^{2}$ D. Liko, ${ }^{2}$ I. Mikulec, ${ }^{2}$ F. M. Pitters, ${ }^{2}$ J. Schieck, ${ }^{2, b}$ R. Schöfbeck, ${ }^{2}$ M. Spanring, ${ }^{2}$ S. Templ, ${ }^{2}$ W. Waltenberger, ${ }^{2}$ C.-E. Wulz, ${ }^{2, b}$ V. Chekhovsky, ${ }^{3}$ A. Litomin, ${ }^{3}$ V. Makarenko, ${ }^{3}$ M. R. Darwish, ${ }^{4, \mathrm{c}}$ E. A. De Wolf, ${ }^{4}$ X. Janssen, ${ }^{4}$ T. Kello, ${ }^{4, d}$ A. Lelek, ${ }^{4}$ H. Rejeb Sfar, ${ }^{4}$ P. Van Mechelen, ${ }^{4}$ S. Van Putte, ${ }^{4}$ N. Van Remortel, ${ }^{4}$ F. Blekman, ${ }^{5}$ E. S. Bols, ${ }^{5}$ J. D'Hondt, ${ }^{5}$ J. De Clercq, ${ }^{5}$ M. Delcourt, ${ }^{5}$ S. Lowette, ${ }^{5}$ S. Moortgat, ${ }^{5}$ A. Morton, ${ }^{5}$ D. Müller, ${ }^{5}$ A. R. Sahasransu, ${ }^{5}$ S. Tavernier, ${ }^{5}$ W. Van Doninck, ${ }^{5}$ P. Van Mulders, ${ }^{5}$ D. Beghin, ${ }^{6}$ B. Bilin, ${ }^{6}$ B. Clerbaux, ${ }^{6}$ G. De Lentdecker, ${ }^{6}$ L. Favart, ${ }^{6}$ A. Grebenyuk, ${ }^{6}$ A. K. Kalsi, ${ }^{6}$ K. Lee, ${ }^{6}$ M. Mahdavikhorrami, ${ }^{6}$ I. Makarenko, ${ }^{6}$ L. Moureaux, ${ }^{6}$ L. Pétré, ${ }^{6}$ A. Popov, ${ }^{6}$ N. Postiau, ${ }^{6}$ E. Starling, ${ }^{6}$ L. Thomas, ${ }^{6}$ M. Vanden Bemden, ${ }^{6}$ C. Vander Velde, ${ }^{6}$ P. Vanlaer, ${ }^{6}$ D. Vannerom, ${ }^{6}$ L. Wezenbeek, ${ }^{6}$ T. Cornelis, ${ }^{7}$ D. Dobur, ${ }^{7}$ M. Gruchala, ${ }^{7}$ L. Lambrecht, ${ }^{7}$ G. Mestdach, ${ }^{7}$ M. Niedziela, ${ }^{7}$ C. Roskas, ${ }^{7}$ K. Skovpen, ${ }^{7}$ T. T. Tran, ${ }^{7}$ M. Tytgat, ${ }^{7}$ W. Verbeke, ${ }^{7}$ B. Vermassen, ${ }^{7}$ M. Vit, ${ }^{7}$ A. Bethani,${ }^{8}$ G. Bruno,${ }^{8}$ F. Bury, ${ }^{8}$ C. Caputo,${ }^{8}$ P. David, ${ }^{8}$ C. Delaere, ${ }^{8}$ I. S. Donertas, ${ }^{8}$ A. Giammanco,${ }^{8}$ K. Jaffel,${ }^{8}$ V. Lemaitre, ${ }^{8}$ K. Mondal,${ }^{8}$ J. Prisciandaro, ${ }^{8}$ A. Taliercio, ${ }^{8}$ M. Teklishyn,${ }^{8}$ P. Vischia,${ }^{8}$ S. Wertz ${ }^{8}$ S. Wuyckens, ${ }^{8}$ G. A. Alves,${ }^{9}$ C. Hensel, ${ }^{9}$ A. Moraes, ${ }^{9}$ W. L. Aldá Júnior,${ }^{10}$ M. Barroso Ferreira Filho, ${ }^{10}$ H. Brandao Malbouisson, ${ }^{10}$ W. Carvalho, ${ }^{10}$ J. Chinellato, ${ }^{10, e}$ E. M. Da Costa, ${ }^{10}$ G. G. Da Silveira, ${ }^{10, \mathrm{f}}$ D. De Jesus Damiao, ${ }^{10}$ S. Fonseca De Souza, ${ }^{10}$

D. Matos Figueiredo, ${ }^{10}$ C. Mora Herrera,${ }^{10}$ K. Mota Amarilo, ${ }^{10}$ L. Mundim, ${ }^{10}$ H. Nogima, ${ }^{10}$ P. Rebello Teles, ${ }^{10}$ L. J. Sanchez Rosas, ${ }^{10}$ A. Santoro, ${ }^{10}$ S. M. Silva Do Amaral, ${ }^{10}$ A. Sznajder, ${ }^{10}$ M. Thiel, ${ }^{10}$ F. Torres Da Silva De Araujo, ${ }^{10}$ 
A. Vilela Pereira, ${ }^{10}$ C. A. Bernardes, ${ }^{11 \mathrm{a}}$ L. Calligaris, ${ }^{11 \mathrm{a}}$ T. R. Fernandez Perez Tomei, ${ }^{11 \mathrm{a}}$ E. M. Gregores, ${ }^{11 \mathrm{a}, 11 \mathrm{~b}}$ D. S. Lemos, ${ }^{11 \mathrm{a}}$ P. G. Mercadante, ${ }^{11 \mathrm{a}, 1 \mathrm{~b}}$ S. F. Novaes, ${ }^{1 \mathrm{a} a}$ Sandra S. Padula, ${ }^{11 \mathrm{a}}$ A. Aleksandrov, ${ }^{12}$ G. Antchev,${ }^{12}$ I. Atanasov, ${ }^{12}$ R. Hadjiiska, ${ }^{12}$ P. Iaydjiev, ${ }^{12}$ M. Misheva, ${ }^{12}$ M. Rodozov, ${ }^{12}$ M. Shopova, ${ }^{12}$ G. Sultanov, ${ }^{12}$ A. Dimitrov, ${ }^{13}$ T. Ivanov, ${ }^{13}$ L. Litov, ${ }^{13}$ B. Pavlov, ${ }^{13}$ P. Petkov, ${ }^{13}$ A. Petrov,${ }^{13}$ T. Cheng, ${ }^{14}$ W. Fang,${ }^{14, d}$ Q. Guo, ${ }^{14}$ T. Javaid,,${ }^{14, g}$ M. Mittal, ${ }^{14}$ H. Wang,${ }^{14}$ L. Yuan, ${ }^{14}$ M. Ahmad, ${ }^{15}$ G. Bauer, ${ }^{15}$ C. Dozen, ${ }^{15, h}$ Z. Hu,${ }^{15}$ J. Martins, ${ }^{15, \mathrm{i}}$ Y. Wang,${ }^{15}$ K. Yi,${ }^{15, j, k}$ E. Chapon, ${ }^{16}$ G. M. Chen, ${ }^{16, g}$ H. S. Chen, ${ }^{16,9}$ M. Chen, ${ }^{16}$ F. Iemmi ${ }^{16}$ A. Kapoor, ${ }^{16}$ D. Leggat ${ }^{16}$ H. Liao, ${ }^{16}$ Z.-A. Liu, ${ }^{16,1}$ R. Sharma, ${ }^{16}$ A. Spiezia, ${ }^{16}$ J. Tao, ${ }^{16}$ J. Thomas-wilsker, ${ }^{16}$ J. Wang, ${ }^{16}$ H. Zhang, ${ }^{16}$ S. Zhang, ${ }^{16, g}$ J. Zhao, ${ }^{16}$ A. Agapitos, ${ }^{17}$ Y. Ban, ${ }^{17}$ C. Chen, ${ }^{17}$ Q. Huang, ${ }^{17}$ A. Levin, ${ }^{17}$ Q. Li,${ }^{17}$ M. Lu,${ }^{17}$ X. Lyu, ${ }^{17}$ Y. Mao, ${ }^{17}$ S. J. Qian, ${ }^{17}$ D. Wang, ${ }^{17}$ Q. Wang, ${ }^{17}$ J. Xiao, ${ }^{17}$ Z. You, ${ }^{18}$ X. Gao, ${ }^{19, d}$ H. Okawa, ${ }^{19}$ M. Xiao, ${ }^{20}$ C. Avila, ${ }^{21}$ A. Cabrera, ${ }^{21}$ C. Florez, ${ }^{21}$ J. Fraga, ${ }^{21}$ A. Sarkar, ${ }^{21}$ M. A. Segura Delgado, ${ }^{21}$ J. Jaramillo, ${ }^{22}$ J. Mejia Guisao, ${ }^{22}$ F. Ramirez, ${ }^{22}$ J. D. Ruiz Alvarez, ${ }^{22}$ C. A. Salazar González, ${ }^{22}$ N. Vanegas Arbelaez, ${ }^{22}$ D. Giljanovic, ${ }^{23}$ N. Godinovic, ${ }^{23}$ D. Lelas, ${ }^{23}$ I. Puljak, ${ }^{23}$ Z. Antunovic, ${ }^{24}$ M. Kovac, ${ }^{24}$ T. Sculac, ${ }^{24}$ V. Brigljevic, ${ }^{25}$ D. Ferencek, ${ }^{25}$ D. Majumder, ${ }^{25}$ M. Roguljic, ${ }^{25}$ A. Starodumov, ${ }^{25, m}$ T. Susa, ${ }^{25}$ A. Attikis, ${ }^{26}$ E. Erodotou, ${ }^{26}$ A. Ioannou, ${ }^{26}$ G. Kole,${ }^{26}$ M. Kolosova, ${ }^{26}$ S. Konstantinou, ${ }^{26}$ J. Mousa ${ }^{26}$ C. Nicolaou, ${ }^{26}$ F. Ptochos,${ }^{26}$ P. A. Razis,${ }^{26}$ H. Rykaczewski, ${ }^{26}$ H. Saka, ${ }^{26}$ M. Finger, ${ }^{27, \mathrm{n}}$ M. Finger Jr., ${ }^{27, \mathrm{n}}$ A. Kveton, ${ }^{27}$ E. Ayala, ${ }^{28}$ E. Carrera Jarrin, ${ }^{29}$ S. Abu Zeid, ${ }^{30, \mathrm{o}}$ S. Khalil, ${ }^{30, p}$ E. Salama, ${ }^{30, \mathrm{q}, \mathrm{o}}$ A. Lotfy, ${ }^{31}$ M. A. Mahmoud ${ }^{31}$ S. Bhowmik, ${ }^{32}$ A. Carvalho Antunes De Oliveira, ${ }^{32}$ R. K. Dewanjee, ${ }^{32}$ K. Ehataht ${ }^{32}$ M. Kadastik, ${ }^{32}$ J. Pata, ${ }^{32}$ M. Raidal, ${ }^{32}$ C. Veelken, ${ }^{32}$ P. Eerola,${ }^{33}$ L. Forthomme,${ }^{33}$ H. Kirschenmann, ${ }^{33}$ K. Osterberg, ${ }^{33}$ M. Voutilainen, ${ }^{33}$ E. Brücken, ${ }^{34}$ F. Garcia, ${ }^{34}$ J. Havukainen, ${ }^{34}$ V. Karimäki, ${ }^{34}$ M. S. Kim, ${ }^{34}$ R. Kinnunen, ${ }^{34}$ T. Lampén, ${ }^{34}$ K. Lassila-Perini, ${ }^{34}$ S. Lehti, ${ }^{34}$ T. Lindén, ${ }^{34}$ M. Lotti, ${ }^{34}$ L. Martikainen, ${ }^{34}$ H. Siikonen,${ }^{34}$ E. Tuominen, ${ }^{34}$ J. Tuominiemi, ${ }^{34}$ P. Luukka, ${ }^{35}$ H. Petrow, ${ }^{35}$ T. Tuuva, ${ }^{35}$ C. Amendola,${ }^{36}$ M. Besancon, ${ }^{36}$ F. Couderc, ${ }^{36}$ M. Dejardin, ${ }^{36}$ D. Denegri, ${ }^{36}$ J. L. Faure ${ }^{36}$ F. Ferri, ${ }^{36}$ S. Ganjour, ${ }^{36}$ A. Givernaud,${ }^{36}$ P. Gras,${ }^{36}$ G. Hamel de Monchenault, ${ }^{36}$ P. Jarry, ${ }^{36}$ B. Lenzi ${ }^{36}$ E. Locci ${ }^{36}$ J. Malcles, ${ }^{36}$ J. Rander, ${ }^{36}$ A. Rosowsky, ${ }^{36}$ M. Ö. Sahin, ${ }^{36}$ A. Savoy-Navarro, ${ }^{36, r}$ M. Titov, ${ }^{36}$ G. B. Yu, ${ }^{36}$ S. Ahuja,${ }^{37}$ F. Beaudette, ${ }^{37}$ M. Bonanomi, ${ }^{37}$ A. Buchot Perraguin, ${ }^{37}$ P. Busson, ${ }^{37}$ A. Cappati, ${ }^{37}$ C. Charlot,${ }^{37}$ O. Davignon, ${ }^{37}$ B. Diab, ${ }^{37}$ G. Falmagne, ${ }^{37}$ S. Ghosh,${ }^{37}$ R. Granier de Cassagnac, ${ }^{37}$ A. Hakimi, ${ }^{37}$ I. Kucher ${ }^{37}$ A. Lobanov,${ }^{37}$ M. Nguyen, ${ }^{37}$ C. Ochando, ${ }^{37}$ P. Paganini, ${ }^{37}$ J. Rembser, ${ }^{37}$ R. Salerno, ${ }^{37}$ J. B. Sauvan, ${ }^{37}$ Y. Sirois, ${ }^{37}$ A. Zabi, ${ }^{37}$ A. Zghiche, ${ }^{37}$ J.-L. Agram, ${ }^{38, \mathrm{~s}}$ J. Andrea, ${ }^{38}$ D. Apparu, ${ }^{38}$ D. Bloch,${ }^{38}$ G. Bourgatte, ${ }^{38}$ J.-M. Brom,${ }^{38}$ E. C. Chabert, ${ }^{38}$ C. Collard, ${ }^{38}$ D. Darej, ${ }^{38}$ J.-C. Fontaine, ${ }^{38, \mathrm{~s}}$ U. Goerlach,${ }^{38}$ C. Grimault, ${ }^{38}$ A.-C. Le Bihan, ${ }^{38}$ P. Van Hove, ${ }^{38}$ E. Asilar, ${ }^{39}$ S. Beauceron, ${ }^{39}$ C. Bernet,${ }^{39}$ G. Boudoul, ${ }^{39}$ C. Camen,${ }^{39}$ A. Carle,${ }^{39}$ N. Chanon, ${ }^{39}$ D. Contardo,${ }^{39}$ P. Depasse, ${ }^{39}$ H. El Mamouni, ${ }^{39}$ J. Fay,${ }^{39}$ S. Gascon, ${ }^{39}$ M. Gouzevitch ${ }^{39}$ B. Ille, ${ }^{39}$ Sa. Jain, ${ }^{39}$ I. B. Laktineh, ${ }^{39}$ H. Lattaud, ${ }^{39}$ A. Lesauvage, ${ }^{39}$ M. Lethuillier, ${ }^{39}$ L. Mirabito, ${ }^{39}$ K. Shchablo, ${ }^{39}$ L. Torterotot, ${ }^{39}$ G. Touquet, ${ }^{39}$ M. Vander Donckt,${ }^{39}$ S. Viret, ${ }^{39}$ I. Lomidze,${ }^{40}$ T. Toriashvili, ${ }^{40, t}$ Z. Tsamalaidze,${ }^{40, \mathrm{~L}}$ L. Feld ${ }^{41}$ K. Klein, ${ }^{41}$ M. Lipinski, ${ }^{41}$ D. Meuser, ${ }^{41}$ A. Pauls,${ }^{41}$ M. P. Rauch, ${ }^{41}$ M. Teroerde,${ }^{41}$ D. Eliseev, ${ }^{42}$ M. Erdmann, ${ }^{42}$ P. Fackeldey, ${ }^{42}$ B. Fischer, ${ }^{42}$ S. Ghosh, ${ }^{42}$ T. Hebbeker, ${ }^{42}$ K. Hoepfner, ${ }^{42}$ F. Ivone, ${ }^{42}$ H. Keller, ${ }^{42}$

L. Mastrolorenzo, ${ }^{42}$ M. Merschmeyer, ${ }^{42}$ A. Meyer ${ }^{42}$ G. Mocellin, ${ }^{42}$ S. Mondal, ${ }^{42}$ S. Mukherjee, ${ }^{42}$ D. Noll,${ }^{42}$ A. Novak, ${ }^{42}$

T. Pook, ${ }^{42}$ A. Pozdnyakov, ${ }^{42}$ Y. Rath,${ }^{42}$ H. Reithler ${ }^{42}$ J. Roemer, ${ }^{42}$ A. Schmidt, ${ }^{42}$ S. C. Schuler,${ }^{42}$ A. Sharma, ${ }^{42}$

S. Wiedenbeck, ${ }^{42}$ S. Zaleski, ${ }^{42}$ C. Dziwok ${ }^{43}$ G. Flügge, ${ }^{43}$ W. Haj Ahmad, ${ }^{43, u}$ O. Hlushchenko, ${ }^{43}$ T. Kress, ${ }^{43}$ A. Nowack,${ }^{43}$ C. Pistone,${ }^{43}$ O. Pooth,${ }^{43}$ D. Roy,${ }^{43}$ H. Sert, ${ }^{43}$ A. Stahl,${ }^{43, v}$ T. Ziemons, ${ }^{43}$ H. Aarup Petersen, ${ }^{44}$ M. Aldaya Martin, ${ }^{44}$ P. Asmuss, ${ }^{44}$ I. Babounikau, ${ }^{44}$ S. Baxter, ${ }^{44}$ O. Behnke, ${ }^{44}$ A. Bermúdez Martínez, ${ }^{44}$ A. A. Bin Anuar, ${ }^{44}$ K. Borras, ${ }^{44, w}$ V. Botta, ${ }^{44}$ D. Brunner, ${ }^{44}$ A. Campbell, ${ }^{44}$ A. Cardini, ${ }^{44}$ C. Cheng, ${ }^{44}$ P. Connor,${ }^{44}$ S. Consuegra Rodríguez, ${ }^{44}$ V. Danilov, ${ }^{44}$ M. M. Defranchis, ${ }^{44}$ L. Didukh, ${ }^{44}$ G. Eckerlin, ${ }^{44}$ D. Eckstein, ${ }^{44}$ L. I. Estevez Banos,${ }^{44}$ O. Filatov,${ }^{44}$ E. Gallo, ${ }^{44, x}$ A. Geiser,${ }^{44}$

A. Giraldi, ${ }^{44}$ A. Grohsjean, ${ }^{44}$ M. Guthoff, ${ }^{44}$ A. Jafari, ${ }^{44, y}$ N. Z. Jomhari, ${ }^{44}$ H. Jung, ${ }^{44}$ A. Kasem, ${ }^{44, w}$ M. Kasemann, ${ }^{44}$ H. Kaveh, ${ }^{44}$ C. Kleinwort, ${ }^{44}$ J. Knolle, ${ }^{44}$ D. Krücker, ${ }^{44}$ W. Lange, ${ }^{44}$ T. Lenz, ${ }^{44}$ J. Lidrych, ${ }^{44}$ K. Lipka, ${ }^{44}$ W. Lohmann, ${ }^{44, z}$

T. Madlener, ${ }^{44}$ R. Mankel, ${ }^{44}$ I.-A. Melzer-Pellmann, ${ }^{44}$ J. Metwally, ${ }^{44}$ A. B. Meyer, ${ }^{44}$ M. Meyer, ${ }^{44}$ J. Mnich, ${ }^{44}$

A. Mussgiller, ${ }^{44}$ V. Myronenko, ${ }^{44}$ Y. Otarid, ${ }^{44}$ D. Pérez Adán, ${ }^{44}$ D. Pitzl,${ }^{44}$ A. Raspereza, ${ }^{44}$ B. Ribeiro Lopes,${ }^{44}$

J. Rübenach, ${ }^{44}$ A. Saggio, ${ }^{44}$ A. Saibel,${ }^{44}$ M. Savitskyi, ${ }^{44}$ V. Scheurer ${ }^{44}$ C. Schwanenberger, ${ }^{44, x}$ A. Singh, ${ }^{44}$

R. E. Sosa Ricardo, ${ }^{44}$ D. Stafford,${ }^{44}$ N. Tonon, ${ }^{44}$ O. Turkot, ${ }^{44}$ A. Vagnerini, ${ }^{44}$ M. Van De Klundert, ${ }^{44}$ R. Walsh,${ }^{44}$ D. Walter, ${ }^{44}$ Y. Wen, ${ }^{44}$ K. Wichmann, ${ }^{44}$ C. Wissing, ${ }^{44}$ S. Wuchterl, ${ }^{44}$ R. Zlebcik, ${ }^{44}$ R. Aggleton, ${ }^{45}$ S. Bein,${ }^{45}$ L. Benato, ${ }^{45}$ A. Benecke, ${ }^{45}$ K. De Leo, ${ }^{45}$ T. Dreyer, ${ }^{45}$ M. Eich ${ }^{45}$ F. Feindt, ${ }^{45}$ A. Fröhlich, ${ }^{45}$ C. Garbers, ${ }^{45}$ E. Garutti, ${ }^{45}$ P. Gunnellini, ${ }^{45}$ J. Haller, ${ }^{45}$

A. Hinzmann, ${ }^{45}$ A. Karavdina, ${ }^{45}$ G. Kasieczka, ${ }^{45}$ R. Klanner, ${ }^{45}$ R. Kogler, ${ }^{45}$ V. Kutzner, ${ }^{45}$ J. Lange, ${ }^{45}$ T. Lange ${ }^{45}$

A. Malara, ${ }^{45}$ A. Nigamova, ${ }^{45}$ K. J. Pena Rodriguez, ${ }^{45}$ O. Rieger, ${ }^{45}$ P. Schleper, ${ }^{45}$ M. Schröder, ${ }^{45}$ J. Schwandt, ${ }^{45}$

D. Schwarz, ${ }^{45}$ J. Sonneveld, ${ }^{45}$ H. Stadie, ${ }^{45}$ G. Steinbrück, ${ }^{45}$ A. Tews, ${ }^{45}$ B. Vormwald, ${ }^{45}$ I. Zoi, ${ }^{45}$ J. Bechtel, ${ }^{46}$ T. Berger, ${ }^{46}$ 
E. Butz, ${ }^{46}$ R. Caspart ${ }^{46}$ T. Chwalek, ${ }^{46}$ W. De Boer ${ }^{46, a}$ A. Dierlamm, ${ }^{46}$ A. Droll,${ }^{46}$ K. El Morabit, ${ }^{46}$ N. Faltermann, ${ }^{46}$ K. Flöh, ${ }^{46}$ M. Giffels, ${ }^{46}$ J. o. Gosewisch, ${ }^{46}$ A. Gottmann, ${ }^{46}$ F. Hartmann, ${ }^{46, v}$ C. Heidecker, ${ }^{46}$ U. Husemann, ${ }^{46}$ I. Katkov, ${ }^{46, a a}$ P. Keicher, ${ }^{46}$ R. Koppenhöfer, ${ }^{46}$ S. Maier, ${ }^{46}$ M. Metzler, ${ }^{46}$ S. Mitra, ${ }^{46}$ Th. Müller, ${ }^{46}$ M. Neukum, ${ }^{46}$ G. Quast, ${ }^{46}$ K. Rabbertz, ${ }^{46}$ J. Rauser, ${ }^{46}$ D. Savoiu, ${ }^{46}$ D. Schäfer, ${ }^{46}$ M. Schnepf, ${ }^{46}$ D. Seith, ${ }^{46}$ I. Shvetsov, ${ }^{46}$ H. J. Simonis, ${ }^{46}$ R. Ulrich, ${ }^{46}$ J. Van Der Linden, ${ }^{46}$ R. F. Von Cube, ${ }^{46}$ M. Wassmer, ${ }^{46}$ M. Weber, ${ }^{46}$ S. Wieland, ${ }^{46}$ R. Wolf,${ }^{46}$ S. Wozniewski, ${ }^{46}$ S. Wunsch,${ }^{46}$ G. Anagnostou, ${ }^{47}$ P. Asenov, ${ }^{47}$ G. Daskalakis, ${ }^{47}$ T. Geralis, ${ }^{47}$ A. Kyriakis, ${ }^{47}$ D. Loukas, ${ }^{47}$ A. Stakia,${ }^{47}$ M. Diamantopoulou, ${ }^{48}$

D. Karasavvas, ${ }^{48}$ G. Karathanasis, ${ }^{48}$ P. Kontaxakis, ${ }^{48}$ C. K. Koraka ${ }^{48}$ A. Manousakis-katsikakis, ${ }^{48}$ A. Panagiotou, ${ }^{48}$ I. Papavergou, ${ }^{48}$ N. Saoulidou, ${ }^{48}$ K. Theofilatos, ${ }^{48}$ E. Tziaferi, ${ }^{48}$ K. Vellidis, ${ }^{48}$ E. Vourliotis, ${ }^{48}$ G. Bakas,${ }^{49}$ K. Kousouris, ${ }^{49}$ I. Papakrivopoulos, ${ }^{49}$ G. Tsipolitis, ${ }^{49}$ A. Zacharopoulou, ${ }^{49}$ I. Evangelou, ${ }^{50}$ C. Foudas,${ }^{50}$ P. Gianneios,${ }^{50}$ P. Katsoulis, ${ }^{50}$ P. Kokkas, ${ }^{50}$ N. Manthos,${ }^{50}$ I. Papadopoulos ${ }^{50}$ J. Strologas,${ }^{50}$ M. Csanad ${ }^{51}$ K. Farkas, ${ }^{51}$ M. M. A. Gadallah,,${ }^{51, b b}$ S. Lökös, ${ }^{51, c c}$ P. Major, ${ }^{51}$ K. Mandal,${ }^{51}$ A. Mehta,${ }^{51}$ G. Pasztor, ${ }^{51}$ A. J. Rádl, ${ }^{51}$ O. Surányi,${ }^{51}$ G. I. Veres, ${ }^{51}$ M. Bartók,${ }^{52, d d}$ G. Bencze, ${ }^{52}$ C. Hajdu, ${ }^{52}$ D. Horvath,${ }^{52, e e}$ F. Sikler, ${ }^{52}$ V. Veszpremi, ${ }^{52}$ G. Vesztergombi, ${ }^{52, a, f f}$ S. Czellar, ${ }^{53}$ J. Karancsi,${ }^{53, d d}$ J. Molnar, ${ }^{53}$ Z. Szillasi, ${ }^{53}$ D. Teyssier, ${ }^{53}$ P. Raics,${ }^{54}$ Z. L. Trocsanyi,,${ }^{54, f f}$ B. Ujvari, ${ }^{54}$ T. Csorgo, ${ }^{55, g g}$ F. Nemes, ${ }^{55, g g}$ T. Novak, ${ }^{55}$ S. Choudhury, ${ }^{56}$ J. R. Komaragiri, ${ }^{56}$ D. Kumar, ${ }^{56}$ L. Panwar, ${ }^{56}$ P. C. Tiwari, ${ }^{56}$ S. Bahinipati,,${ }^{57, \text { hh }}$ D. Dash, ${ }^{57}$ C. Kar, ${ }^{57}$ P. Mal, ${ }^{57}$ T. Mishra, ${ }^{57}$ V. K. Muraleedharan Nair Bindhu, ${ }^{57, \text { ii }}$ A. Nayak, ${ }^{57, \text { ii }}$ P. Saha, ${ }^{57}$ N. Sur, ${ }^{57}$ S. K. Swain, ${ }^{57}$ S. Bansal, ${ }^{58}$ S. B. Beri, ${ }^{58}$ V. Bhatnagar, ${ }^{58}$ G. Chaudhary,${ }^{58}$ S. Chauhan, ${ }^{58}$ N. Dhingra, ${ }^{58, j j}$ R. Gupta, ${ }^{58}$ A. Kaur ${ }^{58}$ S. Kaur, ${ }^{58}$ P. Kumari, ${ }^{58}$ M. Meena, ${ }^{58}$ K. Sandeep, ${ }^{58}$ J. B. Singh, ${ }^{58}$ A. K. Virdi, ${ }^{58}$ A. Ahmed,${ }^{59}$ A. Bhardwaj, ${ }^{59}$ B. C. Choudhary, ${ }^{59}$ R. B. Garg, ${ }^{59}$ M. Gola, ${ }^{59}$ S. Keshri, ${ }^{59}$ A. Kumar, ${ }^{59}$ M. Naimuddin,${ }^{59}$ P. Priyanka,${ }^{59}$ K. Ranjan, ${ }^{59}$ A. Shah, ${ }^{59}$ M. Bharti, ${ }^{60, k k}$ R. Bhattacharya, ${ }^{60}$ S. Bhattacharya, ${ }^{60}$ D. Bhowmik, ${ }^{60}$ S. Dutta, ${ }^{60}$ B. Gomber, ${ }^{60,11}$ M. Maity, ${ }^{60, m m}$ S. Nandan, ${ }^{60}$ P. Palit,${ }^{60}$ P. K. Rout, ${ }^{60}$ G. Saha, ${ }^{60}$ B. Sahu, ${ }^{60}$ S. Sarkar, ${ }^{60}$ M. Sharan, ${ }^{60}$ B. Singh,${ }^{60, k k}$ S. Thakur, ${ }^{60, k k}$ P. K. Behera, ${ }^{61}$ S. C. Behera, ${ }^{61}$ P. Kalbhor, ${ }^{61}$ A. Muhammad, ${ }^{61}$ R. Pradhan, ${ }^{61}$ P. R. Pujahari, ${ }^{61}$ A. Sharma, ${ }^{61}$ A. K. Sikdar, ${ }^{61}$ D. Dutta, ${ }^{62}$ V. Jha, ${ }^{62}$ V. Kumar, ${ }^{62}$

D. K. Mishra, ${ }^{62}$ K. Naskar, ${ }^{62, n n}$ P. K. Netrakanti, ${ }^{62}$ L. M. Pant, ${ }^{62}$ P. Shukla, ${ }^{62}$ T. Aziz ${ }^{63}$ S. Dugad, ${ }^{63}$ M. Kumar, ${ }^{63}$ G. B. Mohanty, ${ }^{63}$ U. Sarkar, ${ }^{63}$ S. Banerjee, ${ }^{64}$ S. Bhattacharya,${ }^{64}$ R. Chudasama, ${ }^{64}$ M. Guchait, ${ }^{64}$ S. Karmakar,${ }^{64}$ S. Kumar,${ }^{64}$ G. Majumder, ${ }^{64}$ K. Mazumdar, ${ }^{64}$ S. Mukherjee,${ }^{64}$ D. Roy, ${ }^{64}$ S. Dube,${ }^{65}$ B. Kansal,${ }^{65}$ S. Pandey, ${ }^{65}$ A. Rane, ${ }^{65}$ A. Rastogi, ${ }^{65}$ S. Sharma, ${ }^{65}$ H. Bakhshiansohi, ${ }^{66,00}$ M. Zeinali, ${ }^{66, p p}$ S. Chenarani, ${ }^{67, q 9}$ S. M. Etesami, ${ }^{67}$ M. Khakzad ${ }^{67}$

M. Mohammadi Najafabadi ${ }^{67}$ M. Felcini, ${ }^{68}$ M. Grunewald, ${ }^{68}$ M. Abbrescia,${ }^{69 a, 69 b}$ R. Aly, ${ }^{69 a, 69 b, r r}$ C. Aruta, ${ }^{69 a, 69 b}$ A. Colaleo, ${ }^{69 \mathrm{a}}$ D. Creanza, ${ }^{69 \mathrm{a}, 69 \mathrm{c}}$ N. De Filippis,${ }^{69 \mathrm{a}, 69 \mathrm{c}}$ M. De Palma ${ }^{69 \mathrm{a}, 69 \mathrm{~b}}$ A. Di Florio, ${ }^{69 \mathrm{a}, 69 \mathrm{~b}}$ A. Di Pilato,${ }^{69 \mathrm{a}, 69 \mathrm{~b}}$ W. Elmetenawee, ${ }^{69 a, 69 b}$ L. Fiore ${ }^{69 a}$ A. Gelmi, ${ }^{69 a, 69 b}$ M. Gul, ${ }^{69 a}$ G. Iaselli, ${ }^{69 a, 69 c}$ M. Ince ${ }^{69 a, 69 b}$ S. Lezki, ${ }^{69 a, 69 b}$ G. Maggi ${ }^{69 a, 69 c}$ M. Maggi, ${ }^{69 a}$ I. Margjeka, ${ }^{69 a, 69 b}$ V. Mastrapasqua ${ }^{69 a, 69 b}$ J. A. Merlin, ${ }^{69 a}$ S. My,${ }^{6 a, 69 b}$ S. Nuzzo, ${ }^{69 a, 69 b}$ A. Pellecchia, ${ }^{69 a, 69 b}$ A. Pompili, ${ }^{69 a, 69 b}$ G. Pugliese ${ }^{69 a, 69 c}$ A. Ranieri, ${ }^{69 a}$ G. Selvaggi,${ }^{69 a, 69 b}$ L. Silvestris,${ }^{69 a}$ F. M. Simone ${ }^{69 a, 69 b}$ R. Venditti, ${ }^{69 a}$ P. Verwilligen, ${ }^{69 \mathrm{a}}$ G. Abbiendi, ${ }^{70 \mathrm{a}}$ C. Battilana, ${ }^{70 \mathrm{a}, 70 \mathrm{~b}}$ D. Bonacorsi, ${ }^{70 \mathrm{a}, 70 \mathrm{~b}}$ L. Borgonovi, ${ }^{70 \mathrm{a}} \mathrm{S}$. Braibant-Giacomelli, ${ }^{70 \mathrm{a}, 70 \mathrm{~b}}$ L. Brigliadori, ${ }^{70 a}$ R. Campanini, ${ }^{70 a, 70 b}$ P. Capiluppi, ${ }^{70 a, 70 b}$ A. Castro, ${ }^{70 a, 70 b}$ F. R. Cavallo, ${ }^{70 a}$ C. Ciocca, ${ }^{70 a}$ M. Cuffiani, ${ }^{70 a, 70 b}$ G. M. Dallavalle, ${ }^{70 a}$ T. Diotalevi, ${ }^{70 a, 70 b}$ F. Fabbri, ${ }^{70 a}$ A. Fanfani, ${ }^{70 a, 70 b}$ P. Giacomelli, ${ }^{70 a}$ L. Giommi, ${ }^{70 a, 70 b}$ C. Grandi, ${ }^{70 a}$ L. Guiducci, ${ }^{70 a, 70 b}$ S. Lo Meo, ${ }^{70 a, s s}$ L. Lunerti, ${ }^{70 a, 70 b}$ S. Marcellini, ${ }^{70 a}$ G. Masetti, ${ }^{70 a}$ F. L. Navarria, ${ }^{70 a, 70 b}$ A. Perrotta, ${ }^{70 a}$ F. Primavera, ${ }^{70 a, 70 b}$ A. M. Rossi, ${ }^{70 a, 70 b}$ T. Rovelli, ${ }^{70 a, 70 b}$ G. P. Siroli, ${ }^{70 a, 70 b}$ N. Tosi, ${ }^{70 a}$ S. Albergo, ${ }^{71 a, 71 b, t t}$ S. Costa, ${ }^{71 a, 71 b, t t}$ A. Di Mattia, ${ }^{71 a}$ R. Potenza, ${ }^{71 a, 71 b}$ A. Tricomi, ${ }^{71 a, 71 b, t t}$ C. Tuve, ${ }^{71 a, 71 b}$ G. Barbagli, ${ }^{72 a}$ A. Cassese, ${ }^{72 a}$ R. Ceccarelli, ${ }^{72 a, 72 b}$ V. Ciulli, ${ }^{72 a, 72 b}$ C. Civinini, ${ }^{72 a}$ R. D'Alessandro, ${ }^{72 a, 72 b}$ F. Fiori, ${ }^{72 a, 72 b}$ E. Focardi, ${ }^{72 a, 72 b}$ G. Latino, ${ }^{72 a, 72 b}$ P. Lenzi, ${ }^{72 a, 72 b}$ M. Lizzo, ${ }^{72 a, 72 b}$ M. Meschini, ${ }^{72 a}$ S. Paoletti, ${ }^{72 a}$ R. Seidita ${ }^{72 a, 72 b}$ G. Sguazzoni, ${ }^{72 a}$ L. Viliani, ${ }^{72 a}$ L. Benussi, ${ }^{73}$ S. Bianco, ${ }^{73}$ D. Piccolo, ${ }^{73}$ M. Bozzo, ${ }^{74 a, 74 b}$ F. Ferro, ${ }^{74 a}$ R. Mulargia ${ }^{74 a, 74 b}$ E. Robutti ${ }^{74 a}$ S. Tosi,${ }^{74 a, 74 b}$ A. Benaglia, ${ }^{75 a}$ F. Brivio, ${ }^{75 a, 75 b}$ F. Cetorelli, ${ }^{75 a, 75 b}$ V. Ciriolo, ${ }^{75 a, 75 b, v}$ F. De Guio, ${ }^{75 a, 75 b}$ M. E. Dinardo, ${ }^{75 a, 75 b}$ P. Dini, ${ }^{75 a}$ S. Gennai, ${ }^{75 a}$ A. Ghezzi, ${ }^{75 a, 75 b}$ P. Govoni, ${ }^{75 a, 75 b}$ L. Guzzi, ${ }^{75 a, 75 b}$ M. Malberti, ${ }^{75 a}$ S. Malvezzi, ${ }^{75 a}$ A. Massironi, ${ }^{75 a}$ D. Menasce, ${ }^{75 a}$ F. Monti, ${ }^{75 a, 75 b}$ L. Moroni, ${ }^{75 a}$ M. Paganoni, ${ }^{75 a, 75 b}$ D. Pedrini, ${ }^{75 a}$ S. Ragazzi,${ }^{75 a, 75 b}$ T. Tabarelli de Fatis, ${ }^{75 a, 75 b}$ D. Valsecchi, ${ }^{75 a, 75 b, v}$ D. Zuolo, ${ }^{75 a, 75 b}$ S. Buontempo, ${ }^{76 a}$ F. Carnevali, ${ }^{76 a, 76 b}$ N. Cavallo, ${ }^{76 a, 76 c}$ A. De Iorio, ${ }^{76 a, 76 b}$ F. Fabozzi, ${ }^{76 a, 76 c}$ A. O. M. Iorio, ${ }^{76 a, 76 b}$ L. Lista, ${ }^{76 a, 76 b}$ S. Meola,${ }^{76 a, 76 d, v}$ P. Paolucci, ${ }^{76 a, v}$ B. Rossi, ${ }^{76 a}$ C. Sciacca, ${ }^{76 a, 76 b}$ P. Azzi, ${ }^{77 a}$ N. Bacchetta, ${ }^{77 a}$ D. Bisello, ${ }^{77 a, 77 b}$ P. Bortignon, ${ }^{77 a}$ A. Bragagnolo, ${ }^{77 a, 77 b}$ R. Carlin, ${ }^{77 a, 77 b}$ P. Checchia, ${ }^{77 a}$

P. De Castro Manzano, ${ }^{77 a}$ T. Dorigo, ${ }^{77 a}$ F. Gasparini ${ }^{77 a, 77 b}$ U. Gasparini, ${ }^{77 a, 77 b}$ S. Y. Hoh,${ }^{77 a, 77 b}$ L. Layer, ${ }^{77, \text {,u }}$ M. Margoni, ${ }^{77 a, 77 b}$ A. T. Meneguzzo, ${ }^{77 a, 77 b}$ M. Presilla, ${ }^{77 a, 77 b}$ P. Ronchese ${ }^{77 a, 77 b}$ R. Rossin, ${ }^{77 a, 77 b}$ F. Simonetto, ${ }^{77 a, 77 b}$ G. Strong, ${ }^{77 a}$ M. Tosi, ${ }^{77 a, 77 b}$ H. Yarar, ${ }^{77 a, 77 b}$ M. Zanetti, ${ }^{77 a, 77 b}$ P. Zotto, ${ }^{77 a, 77 b}$ A. Zucchetta, ${ }^{77 a, 77 b}$ G. Zumerle, ${ }^{77 a, 77 b}$ C. Aime ${ }^{-78 a, 78 b}$ A. Braghieri, ${ }^{78 a}$ S. Calzaferri, ${ }^{78 a, 78 b}$ D. Fiorina, ${ }^{78 a, 78 b}$ P. Montagna, ${ }^{78 a, 78 b}$ S. P. Ratti, ${ }^{78 a, 78 b}$ V. Re, ${ }^{78 a}$ 
M. Ressegotti, ${ }^{78 a, 78 b}$ C. Riccardi, ${ }^{78 a, 78 b}$ P. Salvini, ${ }^{78 a}$ I. Vai, ${ }^{78 a}$ P. Vitulo, ${ }^{78 a, 78 b}$ G. M. Bilei, ${ }^{79 a}$ D. Ciangottini, ${ }^{79 a, 79 b}$ L. Fanò, ${ }^{79 a, 79 b}$ P. Lariccia, ${ }^{79 a, 79 b}$ M. Magherini, ${ }^{79 a, 79 b}$ G. Mantovani, ${ }^{79 a, 79 b}$ V. Mariani, ${ }^{79 a, 79 b}$ M. Menichelli, ${ }^{79 a}$ F. Moscatelli, ${ }^{79 a}$ A. Piccinelli, ${ }^{79 a, 79 b}$ A. Rossi, ${ }^{79 a, 79 b}$ A. Santocchia, ${ }^{79 a, 79 b}$ D. Spiga, ${ }^{79 a}$ T. Tedeschi, ${ }^{79 a, 79 b}$ P. Azzurri, ${ }^{80 a}$ G. Bagliesi, ${ }^{80 \mathrm{a}}$ V. Bertacchi, ${ }^{80 \mathrm{a}, 80 \mathrm{c}}$ L. Bianchini, ${ }^{80 \mathrm{a}}$ T. Boccali, ${ }^{80 \mathrm{a}}$ E. Bossini, ${ }^{80 \mathrm{a}, 80 \mathrm{~b}}$ R. Castaldi, ${ }^{80 \mathrm{a}}$ M. A. Ciocci, ${ }^{80 \mathrm{a}, 80 \mathrm{~b}}$ R. Dell'Orso, ${ }^{80 \mathrm{a}}$ M. R. Di Domenico, ${ }^{80 \mathrm{a}, 80 \mathrm{~d}}$ S. Donato, ${ }^{80 \mathrm{a}}$ A. Giassi, ${ }^{80 \mathrm{a}}$ M. T. Grippo, ${ }^{80 \mathrm{a}}$ F. Ligabue, ${ }^{80 \mathrm{a}, 80 \mathrm{c}}$ E. Manca, ${ }^{80 \mathrm{a}, 80 \mathrm{c}}$ G. Mandorli, ${ }^{80 a, 80 c}$ A. Messineo, ${ }^{80 a, 80 b}$ F. Palla, ${ }^{80 a}$ S. Parolia, ${ }^{80 a, 80 b}$ G. Ramirez-Sanchez, ${ }^{80 a, 80 c}$ A. Rizzi, ${ }^{80 a, 80 b}$ G. Rolandi, ${ }^{80 a, 80 c}$ S. Roy Chowdhury, ${ }^{80 a, 80 c}$ A. Scribano, ${ }^{80 a}$ N. Shafiei, ${ }^{80 a, 80 b}$ P. Spagnolo, ${ }^{80 a}$ R. Tenchini, ${ }^{80 a}$ G. Tonelli, ${ }^{80 a, 80 b}$ N. Turini, ${ }^{80 a, 80 d}$ A. Venturi, ${ }^{80 a}$ P. G. Verdini, ${ }^{80 a}$ M. Campana, ${ }^{81 a, 81 b}$ F. Cavallari, ${ }^{81 a}$ M. Cipriani, ${ }^{81 a, 81 b}$ D. Del Re, ${ }^{81 a, 81 b}$ E. Di Marco, ${ }^{81 \mathrm{a}}$ M. Diemoz, ${ }^{81 \mathrm{a}}$ E. Longo, ${ }^{81 \mathrm{a}, 81 \mathrm{~b}}$ P. Meridiani, ${ }^{81 \mathrm{a}}$ G. Organtini, ${ }^{81 \mathrm{a}, 81 \mathrm{~b}}$ F. Pandolfi, ${ }^{81 \mathrm{a}}$ R. Paramatti, ${ }^{81 \mathrm{a}, 81 \mathrm{~b}}$ C. Quaranta, ${ }^{81 a, 81 b}$ S. Rahatlou, ${ }^{81 a, 81 b}$ C. Rovelli, ${ }^{81 a}$ F. Santanastasio, ${ }^{81 a, 81 b}$ L. Soffi, ${ }^{81 a}$ R. Tramontano, ${ }^{81 a, 81 b}$ N. Amapane, ${ }^{82 a, 82 b}$ R. Arcidiacono, ${ }^{82 a, 82 c}$ S. Argiro, ${ }^{82 a, 82 b}$ M. Arneodo, ${ }^{82 a, 82 c}$ N. Bartosik, ${ }^{82 a}$ R. Bellan, ${ }^{82 a, 82 b}$ A. Bellora, ${ }^{82 a, 82 b}$ J. Berenguer Antequera, ${ }^{82 a, 82 b}$ C. Biino, ${ }^{82 a}$ N. Cartiglia, ${ }^{82 a}$ S. Cometti, ${ }^{82 a}$ M. Costa, ${ }^{82 a, 82 b}$ R. Covarelli, ${ }^{82 a, 82 b}$ N. Demaria, ${ }^{82 a}$ B. Kiani, ${ }^{82 a, 82 b}$ F. Legger, ${ }^{82 a}$ C. Mariotti, ${ }^{82 a}$ S. Maselli, ${ }^{82 a}$ E. Migliore, ${ }^{82 a, 82 b}$ E. Monteil, ${ }^{82 a, 82 b}$ M. Monteno, ${ }^{82 a}$ M. M. Obertino, ${ }^{82 a, 82 b}$ G. Ortona, ${ }^{82 a}$ L. Pacher, ${ }^{82 a, 82 b}$ N. Pastrone, ${ }^{82 a}$ M. Pelliccioni, ${ }^{82 a}$ G. L. Pinna Angioni, ${ }^{82 a, 82 b}$ M. Ruspa, ${ }^{82 a, 82 c}$ R. Salvatico, ${ }^{82 a, 82 b}$ K. Shchelina, ${ }^{82 a, 82 b}$ F. Siviero, ${ }^{82 a, 82 b}$ V. Sola, ${ }^{82 a}$ A. Solano, ${ }^{82 a, 82 b}$ D. Soldi, ${ }^{82 a, 82 b}$ A. Staiano, ${ }^{82 a}$ M. Tornago, ${ }^{82 a, 82 b}$ D. Trocino, ${ }^{82 a, 82 b}$ S. Belforte, ${ }^{83 a}$ V. Candelise, ${ }^{83 a, 83 b}$ M. Casarsa, ${ }^{83 a}$ F. Cossutti, ${ }^{83 a}$ A. Da Rold, ${ }^{83 a, 83 b}$ G. Della Ricca, ${ }^{83 a, 83 b}$ G. Sorrentino, ${ }^{83 a, 83 b}$ F. Vazzoler, ${ }^{83 a, 83 b}$ S. Dogra, ${ }^{84}$ C. Huh, ${ }^{84}$ B. Kim, ${ }^{84}$ D. H. Kim, ${ }^{84}$ G. N. Kim, ${ }^{84}$ J. Kim, ${ }^{84}$ J. Lee, ${ }^{84}$ S. W. Lee, ${ }^{84}$ C. S. Moon, ${ }^{84}$ Y. D. Oh, ${ }^{84}$ S. I. Pak, ${ }^{84}$ B. C. Radburn-Smith, ${ }^{84}$ S. Sekmen, ${ }^{84}$ Y. C. Yang, ${ }^{84}$ H. Kim, ${ }^{85}$ D. H. Moon, ${ }^{85}$ T. J. Kim, ${ }^{86}$ J. Park, ${ }^{86}$ S. Cho, ${ }^{87}$ S. Choi, ${ }^{87}$ Y. Go,${ }^{87}$ B. Hong, ${ }^{87}$ K. Lee,${ }^{87}$ K. S. Lee, ${ }^{87}$ J. Lim, ${ }^{87}$ J. Park, ${ }^{87}$ S. K. Park, ${ }^{87}$ J. Yoo, ${ }^{87}$ J. Goh, ${ }^{88}$ A. Gurtu, ${ }^{88}$ H. S. Kim, ${ }^{89}$ Y. Kim, ${ }^{89}$ J. Almond, ${ }^{90}$ J. H. Bhyun, ${ }^{90}$ J. Choi, ${ }^{90}$ S. Jeon, ${ }^{90}$ J. Kim $^{90}$ J. S. Kim, ${ }^{90}$ S. Ko, ${ }^{90}$ H. Kwon, ${ }^{90}$ H. Lee, ${ }^{90}$ S. Lee, ${ }^{90}$ B. H. Oh, ${ }^{90}$ M. Oh, ${ }^{90}$ S. B. Oh, ${ }^{90}$ H. Seo, ${ }^{90}$ U. K. Yang, ${ }^{90}$ I. Yoon, ${ }^{90}$ D. Jeon, ${ }^{91}$ J. H. Kim, ${ }^{91}$ B. Ko, ${ }^{91}$ J. S. H. Lee, ${ }^{91}$ I. C. Park, ${ }^{91}$ Y. Roh, ${ }^{91}$ D. Song, ${ }^{91}$ I. J. Watson, ${ }^{91}$ S. Ha, ${ }^{92}$ H. D. Yoo, ${ }^{92}$ Y. Choi, ${ }^{93}$ Y. Jeong, ${ }^{93}$ H. Lee, ${ }^{93}$ Y. Lee, ${ }^{93}$ I. Yu, ${ }^{93}$ T. Beyrouthy, ${ }^{94}$ Y. Maghrbi, ${ }^{94}$ V. Veckalns, ${ }^{95, v v}$ M. Ambrozas, ${ }^{96}$ A. Juodagalvis, ${ }^{96}$ A. Rinkevicius, ${ }^{96}$ G. Tamulaitis, ${ }^{96}$ A. Vaitkevicius, ${ }^{96}$ N. Bin Norjoharuddeen, ${ }^{97}$ W. A. T. Wan Abdullah, ${ }^{97}$ M. N. Yusli, ${ }^{97}$ Z. Zolkapli, ${ }^{97}$ J. F. Benitez, ${ }^{98}$ A. Castaneda Hernandez, ${ }^{98}$ J. A. Murillo Quijada, ${ }^{98}$ L. Valencia Palomo, ${ }^{98}$ G. Ayala, ${ }^{99}$ H. Castilla-Valdez, ${ }^{99}$ E. De La Cruz-Burelo, ${ }^{99}$ I. Heredia-De La Cruz, ${ }^{99, w w}$

R. Lopez-Fernandez, ${ }^{99}$ C. A. Mondragon Herrera, ${ }^{99}$ D. A. Perez Navarro, ${ }^{99}$ A. Sanchez-Hernandez, ${ }^{99}$ S. Carrillo Moreno, ${ }^{100}$ C. Oropeza Barrera, ${ }^{100}$ M. Ramirez-Garcia, ${ }^{100}$ F. Vazquez Valencia, ${ }^{100}$ I. Pedraza, ${ }^{101}$ H. A. Salazar Ibarguen, ${ }^{101}$

C. Uribe Estrada, ${ }^{101}$ J. Mijuskovic, ${ }^{102, x x}$ N. Raicevic, ${ }^{102}$ D. Krofcheck, ${ }^{103}$ S. Bheesette, ${ }^{104}$ P. H. Butler, ${ }^{104}$ A. Ahmad, ${ }^{105}$ M. I. Asghar, ${ }^{105}$ A. Awais, ${ }^{105}$ M. I. M. Awan, ${ }^{105}$ H. R. Hoorani, ${ }^{105}$ W. A. Khan, ${ }^{105}$ M. A. Shah, ${ }^{105}$ M. Shoaib, ${ }^{105}$ M. Waqas, ${ }^{105}$ V. Avati, ${ }^{106}$ L. Grzanka, ${ }^{106}$ M. Malawski, ${ }^{106}$ H. Bialkowska, ${ }^{107}$ M. Bluj, ${ }^{107}$ B. Boimska, ${ }^{107}$ T. Frueboes, ${ }^{107}$ M. Górski, ${ }^{107}$ M. Kazana, ${ }^{107}$ M. Szleper, ${ }^{107}$ P. Traczyk, ${ }^{107}$ P. Zalewski, ${ }^{107}$ K. Bunkowski, ${ }^{108}$ K. Doroba, ${ }^{108}$ A. Kalinowski, ${ }^{108}$ M. Konecki, ${ }^{108}$ J. Krolikowski, ${ }^{108}$ M. Walczak, ${ }^{108}$ M. Araujo, ${ }^{109}$ P. Bargassa, ${ }^{109}$ D. Bastos, ${ }^{109}$ A. Boletti, ${ }^{109}$ P. Faccioli, ${ }^{109}$ M. Gallinaro, ${ }^{109}$ J. Hollar, ${ }^{109}$ N. Leonardo, ${ }^{109}$ T. Niknejad, ${ }^{109}$ M. Pisano, ${ }^{109}$ J. Seixas, ${ }^{109}$ O. Toldaiev, ${ }^{109}$ J. Varela, ${ }^{109}$

S. Afanasiev, ${ }^{110}$ D. Budkouski, ${ }^{110}$ P. Bunin, ${ }^{110}$ M. Gavrilenko, ${ }^{110}$ I. Golutvin, ${ }^{110}$ I. Gorbunov, ${ }^{110}$ A. Kamenev, ${ }^{110}$ V. Karjavine, ${ }^{110}$ A. Lanev, ${ }^{110}$ A. Malakhov, ${ }^{110}$ V. Matveev, ${ }^{110, y y, z z}$ V. Palichik, ${ }^{110}$ V. Perelygin, ${ }^{110}$ M. Savina, ${ }^{110}$ D. Seitova, ${ }^{110}$ V. Shalaev, ${ }^{110}$ S. Shmatov, ${ }^{110}$ S. Shulha, ${ }^{110}$ V. Smirnov, ${ }^{110}$ O. Teryaev, ${ }^{110}$ N. Voytishin, ${ }^{110}$ A. Zarubin, ${ }^{110}$ I. Zhizhin, ${ }^{110}$ G. Gavrilov, ${ }^{111}$ V. Golovtcov, ${ }^{111}$ Y. Ivanov, ${ }^{111}$ V. Kim, ${ }^{111, \text { aaa }}$ E. Kuznetsova, ${ }^{111, b b b}$ V. Murzin, ${ }^{111}$ V. Oreshkin, ${ }^{111}$ I. Smirnov, ${ }^{111}$ D. Sosnov, ${ }^{11}$ V. Sulimov, ${ }^{111}$ L. Uvarov, ${ }^{111}$ S. Volkov, ${ }^{111}$ A. Vorobyev, ${ }^{111}$ Yu. Andreev, ${ }^{112}$ A. Dermenev, ${ }^{112}$ S. Gninenko, ${ }^{112}$ N. Golubev, ${ }^{112}$ A. Karneyeu, ${ }^{112}$ M. Kirsanov, ${ }^{112}$ N. Krasnikov, ${ }^{112}$ A. Pashenkov, ${ }^{112}$ G. Pivovarov, ${ }^{112}$ D. Tlisov, ${ }^{112, a}$ A. Toropin, ${ }^{112}$ V. Epshteyn, ${ }^{113}$ V. Gavrilov, ${ }^{113}$ N. Lychkovskaya, ${ }^{113}$ A. Nikitenko, ${ }^{113, c c c}$ V. Popov, ${ }^{113}$ G. Safronov, ${ }^{113}$ A. Spiridonov, ${ }^{113}$ A. Stepennov, ${ }^{113}$ M. Toms, ${ }^{113}$ E. Vlasov, ${ }^{113}$ A. Zhokin, ${ }^{113}$ T. Aushev, ${ }^{114}$ K. Ivanov, ${ }^{114}$ R. Chistov, ${ }^{115, \text { ddd }}$ M. Danilov, ${ }^{115, \text { eee }}$ A. Oskin, ${ }^{115}$ P. Parygin, ${ }^{115}$ S. Polikarpov, ${ }^{115 \text {,eee } \text { V. Andreev, }}{ }^{116}$ M. Azarkin, ${ }^{116}$

I. Dremin, ${ }^{116}$ M. Kirakosyan, ${ }^{116}$ A. Terkulov, ${ }^{116}$ A. Belyaev, ${ }^{117}$ E. Boos, ${ }^{117}$ M. Dubinin, ${ }^{117, f f f}$ L. Dudko, ${ }^{117}$ A. Ershov, ${ }^{117}$ A. Gribushin, ${ }^{117}$ V. Klyukhin, ${ }^{117}$ O. Kodolova, ${ }^{117}$ I. Lokhtin, ${ }^{117}$ S. Obraztsov, ${ }^{117}$ S. Petrushanko, ${ }^{117}$ V. Savrin, ${ }^{117}$ A. Snigirev, ${ }^{117}$ V. Blinov, ${ }^{118, g g g}$ T. Dimova, ${ }^{118, g g g}$ L. Kardapoltsev, ${ }^{118, g g g}$ I. Ovtin, ${ }^{18, g g g}$ Y. Skovpen, ${ }^{118, g g g}$ I. Azhgirey, ${ }^{119}$ I. Bayshev, ${ }^{119}$ V. Kachanov, ${ }^{19}$ A. Kalinin, ${ }^{119}$ D. Konstantinov, ${ }^{119}$ V. Petrov, ${ }^{119}$ R. Ryutin, ${ }^{119}$ A. Sobol, ${ }^{119}$ S. Troshin, ${ }^{119}$ N. Tyurin, ${ }^{119}$ A. Uzunian, ${ }^{119}$ A. Volkov, ${ }^{119}$ A. Babaev, ${ }^{120}$ V. Okhotnikov, ${ }^{120}$ L. Sukhikh, ${ }^{120}$ V. Borchsh, ${ }^{121}$ V. Ivanchenko, ${ }^{121}$

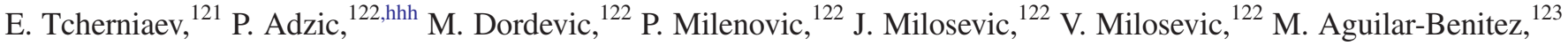


J. Alcaraz Maestre, ${ }^{123}$ A. Álvarez Fernández, ${ }^{123}$ I. Bachiller ${ }^{123}$ M. Barrio Luna, ${ }^{123}$ Cristina F. Bedoya, ${ }^{123}$ C. A. Carrillo Montoya, ${ }^{123}$ M. Cepeda,${ }^{123}$ M. Cerrada, ${ }^{123}$ N. Colino, ${ }^{123}$ B. De La Cruz, ${ }^{123}$ A. Delgado Peris, ${ }^{123}$ J. P. Fernández Ramos, ${ }^{123}$ J. Flix, ${ }^{123}$ M. C. Fouz, ${ }^{123}$ O. Gonzalez Lopez, ${ }^{123}$ S. Goy Lopez, ${ }^{123}$ J. M. Hernandez, ${ }^{123}$ M. I. Josa, ${ }^{123}$ J. León Holgado, ${ }^{123}$ D. Moran, ${ }^{123}$ Á. Navarro Tobar, ${ }^{123}$ A. Pérez-Calero Yzquierdo, ${ }^{123}$ J. Puerta Pelayo, ${ }^{123}$ I. Redondo, ${ }^{123}$ L. Romero, ${ }^{123}$ S. Sánchez Navas, ${ }^{123}$ M. S. Soares, ${ }^{123}$ L. Urda Gómez, ${ }^{123}$ C. Willmott, ${ }^{123}$ J. F. de Trocóniz, ${ }^{124}$ R. Reyes-Almanza, ${ }^{124}$ B. Alvarez Gonzalez, ${ }^{125}$ J. Cuevas, ${ }^{125}$ C. Erice, ${ }^{125}$ J. Fernandez Menendez, ${ }^{125}$ S. Folgueras, ${ }^{125}$

I. Gonzalez Caballero, ${ }^{125}$ E. Palencia Cortezon, ${ }^{125}$ C. Ramón Álvarez,${ }^{125}$ J. Ripoll Sau, ${ }^{125}$ V. Rodríguez Bouza, ${ }^{125}$ A. Trapote, ${ }^{125}$ J. A. Brochero Cifuentes, ${ }^{126}$ I. J. Cabrillo, ${ }^{126}$ A. Calderon, ${ }^{126}$ B. Chazin Quero, ${ }^{126}$ J. Duarte Campderros, ${ }^{126}$ M. Fernandez, ${ }^{126}$ C. Fernandez Madrazo, ${ }^{126}$ P. J. Fernández Manteca, ${ }^{126}$ A. García Alonso, ${ }^{126}$ G. Gomez, ${ }^{126}$ C. Martinez Rivero, ${ }^{126}$ P. Martinez Ruiz del Arbol, ${ }^{126}$ F. Matorras, ${ }^{126}$ P. Matorras Cuevas, ${ }^{126}$ J. Piedra Gomez, ${ }^{126}$ C. Prieels, ${ }^{126}$ F. Ricci-Tam, ${ }^{126}$ T. Rodrigo, ${ }^{126}$ A. Ruiz-Jimeno, ${ }^{126}$ L. Scodellaro, ${ }^{126}$ N. Trevisani, ${ }^{126}$ I. Vila, ${ }^{126}$ J. M. Vizan Garcia, ${ }^{126}$ MK Jayananda, ${ }^{127}$ B. Kailasapathy, ${ }^{127, \text { iii }}$ D. U. J. Sonnadara, ${ }^{127}$ D. D. C. Wickramarathna, ${ }^{127}$ W. G. D. Dharmaratna, ${ }^{128}$ K. Liyanage, ${ }^{128}$ N. Perera, ${ }^{128}$ N. Wickramage, ${ }^{128}$ T. K. Aarrestad, ${ }^{129}$ D. Abbaneo, ${ }^{129}$ J. Alimena, ${ }^{129}$ E. Auffray, ${ }^{129}$ G. Auzinger, ${ }^{129}$ J. Baechler, ${ }^{129}$ P. Baillon, ${ }^{129, a}$ A. H. Ball, ${ }^{129}$ D. Barney, ${ }^{129}$ J. Bendavid ${ }^{129}$ N. Beni, ${ }^{129}$ M. Bianco, ${ }^{129}$ A. Bocci, ${ }^{129}$ E. Brondolin, ${ }^{129}$ T. Camporesi, ${ }^{129}$ M. Capeans Garrido, ${ }^{129}$ G. Cerminara, ${ }^{129}$ S. S. Chhibra, ${ }^{129}$ L. Cristella, ${ }^{129}$ D. d'Enterria, ${ }^{129}$ A. Dabrowski, ${ }^{129}$ N. Daci ${ }^{129}$ A. David, ${ }^{129}$ A. De Roeck, ${ }^{129}$ M. Deile, ${ }^{129}$ R. Di Maria, ${ }^{129}$ M. Dobson, ${ }^{129}$ M. Dünser, ${ }^{129}$ N. Dupont, ${ }^{129}$ A. Elliott-Peisert, ${ }^{129}$ N. Emriskova, ${ }^{129}$ F. Fallavollita, ${ }^{129, j j j}$ D. Fasanella, ${ }^{129}$ S. Fiorendi, ${ }^{129}$ A. Florent, ${ }^{129}$ G. Franzoni, ${ }^{129}$ J. Fulcher, ${ }^{129}$ W. Funk, ${ }^{129}$ S. Giani, ${ }^{129}$ D. Gigi, ${ }^{129}$ K. Gill, ${ }^{129}$ F. Glege, ${ }^{129}$ L. Gouskos, ${ }^{129}$ M. Haranko, ${ }^{129}$ J. Hegeman, ${ }^{129}$ Y. Iiyama, ${ }^{129}$ V. Innocente, ${ }^{129}$ T. James, ${ }^{129}$ P. Janot, ${ }^{129}$ J. Kaspar, ${ }^{129}$ J. Kieseler, ${ }^{129}$ M. Komm, ${ }^{129}$ N. Kratochwil, ${ }^{129}$ C. Lange, ${ }^{129}$ S. Laurila ${ }^{129}$ P. Lecoq, ${ }^{129}$ K. Long, ${ }^{129}$ C. Lourenço, ${ }^{129}$ L. Malgeri, ${ }^{129}$ S. Mallios, ${ }^{129}$ M. Mannelli, ${ }^{129}$ F. Meijers, ${ }^{129}$ S. Mersi, ${ }^{129}$ E. Meschi, ${ }^{129}$ F. Moortgat, ${ }^{129}$ M. Mulders ${ }^{129}$ S. Orfanelli, ${ }^{129}$ L. Orsini,${ }^{129}$ F. Pantaleo, ${ }^{129}$ L. Pape, ${ }^{129}$ E. Perez,${ }^{129}$ M. Peruzzi, ${ }^{129}$ A. Petrilli, ${ }^{129}$ G. Petrucciani, ${ }^{129}$ A. Pfeiffer, ${ }^{129}$ M. Pierini, ${ }^{129}$ M. Pitt, ${ }^{129}$ H. Qu, ${ }^{129}$ T. Quast, ${ }^{129}$ D. Rabady, ${ }^{129}$ A. Racz, ${ }^{129}$ M. Rieger, ${ }^{129}$ M. Rovere, ${ }^{129}$ H. Sakulin, ${ }^{129}$ J. Salfeld-Nebgen, ${ }^{129}$ S. Scarfi, ${ }^{129}$ C. Schäfer,${ }^{129}$ C. Schwick, ${ }^{129}$ M. Selvaggi, ${ }^{129}$ A. Sharma, ${ }^{129}$ P. Silva, ${ }^{129}$ W. Snoeys, ${ }^{129}$ P. Sphicas, ${ }^{129, k k k}$ S. Summers, ${ }^{129}$ V. R. Tavolaro, ${ }^{129}$ D. Treille, ${ }^{129}$ A. Tsirou, ${ }^{129}$ G. P. Van Onsem, ${ }^{129}$ M. Verzetti, ${ }^{129}$ J. Wanczyk, ${ }^{129,111}$ K. A. Wozniak, ${ }^{129}$ W. D. Zeuner, ${ }^{129}$ L. Caminada, ${ }^{130, m m m}$ A. Ebrahimi, ${ }^{130}$ W. Erdmann, ${ }^{130}$ R. Horisberger, ${ }^{130}$ Q. Ingram, ${ }^{130}$ H. C. Kaestli, ${ }^{130}$ D. Kotlinski, ${ }^{130}$ U. Langenegger, ${ }^{130}$ M. Missiroli, ${ }^{130}$ T. Rohe, ${ }^{130}$ K. Androsov,${ }^{131,111}$ M. Backhaus, ${ }^{131}$ P. Berger ${ }^{131}$ A. Calandri, ${ }^{131}$ N. Chernyavskaya, ${ }^{131}$ A. De Cosa, ${ }^{131}$ G. Dissertori, ${ }^{131}$ M. Dittmar, ${ }^{131}$ M. Donegà, ${ }^{131}$ C. Dorfer ${ }^{131}$ F. Eble, ${ }^{131}$ T. Gadek, ${ }^{131}$ T. A. Gómez Espinosa, ${ }^{131}$ C. Grab, ${ }^{131}$ D. Hits, ${ }^{131}$

W. Lustermann, ${ }^{131}$ A.-M. Lyon, ${ }^{131}$ R. A. Manzoni, ${ }^{131}$ C. Martin Perez, ${ }^{131}$ M. T. Meinhard, ${ }^{131}$ F. Micheli, ${ }^{131}$

F. Nessi-Tedaldi, ${ }^{131}$ J. Niedziela ${ }^{131}$ F. Pauss, ${ }^{131}$ V. Perovic, ${ }^{131}$ G. Perrin, ${ }^{131}$ S. Pigazzini, ${ }^{131}$ M. G. Ratti, ${ }^{131}$ M. Reichmann, ${ }^{131}$ C. Reissel, ${ }^{131}$ T. Reitenspiess, ${ }^{131}$ B. Ristic, ${ }^{131}$ D. Ruini, ${ }^{131}$ D. A. Sanz Becerra, ${ }^{131}$ M. Schönenberger, ${ }^{131}$ V. Stampf, ${ }^{131}$

J. Steggemann, ${ }^{131,111}$ R. Wallny, ${ }^{131}$ D. H. Zhu, ${ }^{131}$ C. Amsler, ${ }^{132, n n n}$ P. Bärtschi, ${ }^{132}$ C. Botta, ${ }^{132}$ D. Brzhechko, ${ }^{132}$ M. F. Canelli, ${ }^{132}$ A. De Wit, ${ }^{132}$ R. Del Burgo, ${ }^{132}$ J. K. Heikkilä, ${ }^{132}$ M. Huwiler, ${ }^{132}$ A. Jofrehei, ${ }^{132}$ B. Kilminster, ${ }^{132}$ S. Leontsinis, ${ }^{132}$ A. Macchiolo, ${ }^{132}$ P. Meiring, ${ }^{132}$ V. M. Mikuni, ${ }^{132}$ U. Molinatti, ${ }^{132}$ I. Neutelings,${ }^{132}$ G. Rauco, ${ }^{132}$ A. Reimers, ${ }^{132}$ P. Robmann, ${ }^{132}$ S. Sanchez Cruz, ${ }^{132}$ K. Schweiger, ${ }^{132}$ Y. Takahashi, ${ }^{132}$ C. Adloff, ${ }^{133,000}$ C. M. Kuo, ${ }^{133}$ W. Lin, ${ }^{133}$ A. Roy, ${ }^{133}$ T. Sarkar, ${ }^{133, m m}$ S. S. Yu, ${ }^{133}$ L. Ceard, ${ }^{134}$ P. Chang, ${ }^{134}$ Y. Chao, ${ }^{134}$ K. F. Chen, ${ }^{134}$ P. H. Chen, ${ }^{134}$ W.-S. Hou ${ }^{134}$ Y. y. Li ${ }^{134}$ R.-S. Lu, ${ }^{134}$ E. Paganis, ${ }^{134}$ A. Psallidas, ${ }^{134}$ A. Steen, ${ }^{134}$ E. Yazgan, ${ }^{134}$ P. r. Yu, ${ }^{134}$ B. Asavapibhop, ${ }^{135}$ C. Asawatangtrakuldee, ${ }^{135}$ N. Srimanobhas, ${ }^{135}$ F. Boran, ${ }^{136}$ S. Damarseckin, ${ }^{136, p p p}$ Z. S. Demiroglu, ${ }^{136}$ F. Dolek, ${ }^{136}$ I. Dumanoglu, ${ }^{136, q q q}$ E. Eskut, ${ }^{136}$ G. Gokbulut, ${ }^{136}$ Y. Guler, ${ }^{136}$ E. Gurpinar Guler, ${ }^{136, \text { rrr }}$ I. Hos, ${ }^{136, \text { sss }}$ C. Isik, ${ }^{136}$

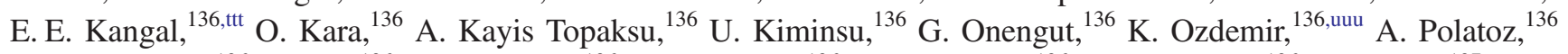
A. E. Simsek, ${ }^{136}$ B. Tali, ${ }^{136, \text { vvv }}$ U. G. Tok, ${ }^{136}$ S. Turkcapar, ${ }^{136}$ I. S. Zorbakir, ${ }^{136}$ C. Zorbilmez, ${ }^{136}$ B. Isildak, ${ }^{137, \text { www }}$ G. Karapinar, ${ }^{137, \mathrm{xxx}}$ K. Ocalan, ${ }^{137, y y y}$ M. Yalvac, ${ }^{137, z z z}$ B. Akgun, ${ }^{138}$ I. O. Atakisi, ${ }^{138}$ E. Gülmez, ${ }^{138}$ M. Kaya, ${ }^{138, \text { aaaa }}$ O. Kaya, ${ }^{138, \text { bbbb }}$ Ö. Özçelik, ${ }^{138}$ S. Tekten,${ }^{138, \text { cccc }}$ E. A. Yetkin, ${ }^{138, \text { dddd }}$ A. Cakir, ${ }^{139}$ K. Cankocak, ${ }^{139, q q q}$ Y. Komurcu, ${ }^{139}$ S. Sen, ${ }^{139, \text { eeee }}$ F. Aydogmus Sen, ${ }^{140}$ S. Cerci, ${ }^{140, v v v}$ B. Kaynak, ${ }^{140}$ S. Ozkorucuklu, ${ }^{140}$ D. Sunar Cerci, ${ }^{140, v v v}$ B. Grynyov, ${ }^{141}$ L. Levchuk, ${ }^{142}$ D. Anthony, ${ }^{143}$ E. Bhal,${ }^{143}$ S. Bologna, ${ }^{143}$ J. J. Brooke, ${ }^{143}$ A. Bundock,${ }^{143}$ E. Clement,${ }^{143}$ D. Cussans,${ }^{143}$ H. Flacher, ${ }^{143}$ J. Goldstein, ${ }^{143}$ G. P. Heath,${ }^{143}$ H. F. Heath, ${ }^{143}$ L. Kreczko, ${ }^{143}$ B. Krikler, ${ }^{143}$ S. Paramesvaran, ${ }^{143}$ T. Sakuma, ${ }^{143}$ S. Seif El Nasr-Storey, ${ }^{143}$ V. J. Smith, ${ }^{143}$ N. Stylianou, ${ }^{143, f f f r}$ J. Taylor, ${ }^{143}$ A. Titterton, ${ }^{143}$ R. White, ${ }^{143}$ K. W. Bell, ${ }^{144}$ A. Belyaev, ${ }^{144, \text { gggg }}$ C. Brew, ${ }^{144}$ R. M. Brown, ${ }^{144}$ D. J. A. Cockerill, ${ }^{144}$ K. V. Ellis, ${ }^{144}$ K. Harder,${ }^{144}$ S. Harper,${ }^{144}$ J. Linacre, ${ }^{144}$ 
K. Manolopoulos, ${ }^{144}$ D. M. Newbold, ${ }^{144}$ E. Olaiya, ${ }^{144}$ D. Petyt, ${ }^{144}$ T. Reis, ${ }^{144}$ T. Schuh, ${ }^{144}$ C. H. Shepherd-Themistocleous, ${ }^{144}$ A. Thea, ${ }^{144}$ I. R. Tomalin, ${ }^{144}$ T. Williams, ${ }^{144}$ R. Bainbridge, ${ }^{145}$ P. Bloch,${ }^{145}$ S. Bonomally, ${ }^{145}$ J. Borg, ${ }^{145}$ S. Breeze, ${ }^{145}$ O. Buchmuller, ${ }^{145}$ V. Cepaitis, ${ }^{145}$ G. S. Chahal, ${ }^{145, \text { hhhh }}$ D. Colling, ${ }^{145}$ P. Dauncey,${ }^{145}$ G. Davies ${ }^{145}$ M. Della Negra, ${ }^{145}$ S. Fayer, ${ }^{145}$ G. Fedi, ${ }^{145}$ G. Hall,${ }^{145}$ M. H. Hassanshahi, ${ }^{145}$ G. Iles, ${ }^{145}$ J. Langford, ${ }^{145}$ L. Lyons, ${ }^{145}$ A.-M. Magnan, ${ }^{145}$ S. Malik, ${ }^{145}$ A. Martelli, ${ }^{145}$ J. Nash, ${ }^{145, \text { iiii }}$ V. Palladino, ${ }^{145}$ M. Pesaresi, ${ }^{145}$ D. M. Raymond, ${ }^{145}$ A. Richards, ${ }^{145}$ A. Rose,${ }^{145}$ E. Scott,${ }^{145}$ C. Seez, ${ }^{145}$ A. Shtipliyski, ${ }^{145}$ A. Tapper, ${ }^{145}$ K. Uchida, ${ }^{145}$ T. Virdee, ${ }^{145, v}$ N. Wardle, ${ }^{145}$ S. N. Webb,${ }^{145}$ D. Winterbottom, ${ }^{145}$ A. G. Zecchinelli, ${ }^{145}$ K. Coldham, ${ }^{146}$ J. E. Cole, ${ }^{146}$ A. Khan, ${ }^{146}$ P. Kyberd, ${ }^{146}$ C. K. Mackay, ${ }^{146}$ I. D. Reid, ${ }^{146}$ L. Teodorescu, ${ }^{146}$ S. Zahid, ${ }^{146}$ S. Abdullin, ${ }^{147}$ A. Brinkerhoff, ${ }^{147}$ B. Caraway, ${ }^{147}$ J. Dittmann, ${ }^{147}$ K. Hatakeyama, ${ }^{147}$ A. R. Kanuganti,${ }^{147}$ B. McMaster, ${ }^{147}$ N. Pastika, ${ }^{147}$ S. Sawant, ${ }^{147}$ C. Smith, ${ }^{147}$ C. Sutantawibul, ${ }^{147}$ J. Wilson, ${ }^{147}$ R. Bartek, ${ }^{148}$ A. Dominguez, ${ }^{148}$ R. Uniyal, ${ }^{148}$ A. M. Vargas Hernandez, ${ }^{148}$ A. Buccilli, ${ }^{149}$ O. Charaf,${ }^{149}$ S. I. Cooper, ${ }^{149}$ D. Di Croce, ${ }^{149}$ S. V. Gleyzer, ${ }^{149}$ C. Henderson, ${ }^{149}$ C. U. Perez,${ }^{149}$ P. Rumerio, ${ }^{149, j j j \mathrm{C}}$ C. West, ${ }^{149}$ A. Akpinar ${ }^{150}$ A. Albert, ${ }^{150}$ D. Arcaro, ${ }^{150}$ C. Cosby, ${ }^{150}$ Z. Demiragli, ${ }^{150}$ E. Fontanesi, ${ }^{150}$ D. Gastler, ${ }^{150}$ J. Rohlf, ${ }^{150}$ K. Salyer, ${ }^{150}$ D. Sperka, ${ }^{150}$ D. Spitzbart, ${ }^{150}$ I. Suarez,${ }^{150}$ A. Tsatsos, ${ }^{150}$ S. Yuan, ${ }^{150}$ D. Zou, ${ }^{150}$ G. Benelli, ${ }^{151}$ B. Burkle, ${ }^{151}$ X. Coubez,${ }^{151, w}$ D. Cutts, ${ }^{151}$ Y. t. Duh, ${ }^{151}$ M. Hadley, ${ }^{151}$ U. Heintz, ${ }^{151}$ J. M. Hogan, ${ }^{151, k k k k}$ E. Laird ${ }^{151}$ G. Landsberg, ${ }^{151}$ K. T. Lau, ${ }^{151}$ J. Lee, ${ }^{151}$ J. Luo, ${ }^{151}$ M. Narain, ${ }^{151}$ S. Sagir, ${ }^{151,1111}$ E. Usai, ${ }^{151}$ W. Y. Wong, ${ }^{151}$ X. Yan, ${ }^{151}$ D. Yu, ${ }^{151}$ W. Zhang, ${ }^{151}$ C. Brainerd, ${ }^{152}$ R. Breedon, ${ }^{152}$ M. Calderon De La Barca Sanchez, ${ }^{152}$ M. Chertok, ${ }^{152}$ J. Conway, ${ }^{152}$ P. T. Cox, ${ }^{152}$ R. Erbacher, ${ }^{152}$ G. Haza, ${ }^{152}$ F. Jensen, ${ }^{152}$ O. Kukral, ${ }^{152}$ R. Lander, ${ }^{152}$ M. Mulhearn, ${ }^{152}$ D. Pellett, ${ }^{152}$ B. Regnery ${ }^{152}$ D. Taylor, ${ }^{152}$ M. Tripathi, ${ }^{152}$ Y. Yao, ${ }^{152}$ F. Zhang, ${ }^{152}$ M. Bachtis, ${ }^{153}$ R. Cousins,${ }^{153}$ A. Dasgupta, ${ }^{153}$ A. Datta, ${ }^{153}$ D. Hamilton, ${ }^{153}$ J. Hauser ${ }^{153}$ M. Ignatenko, ${ }^{153}$ M. A. Iqbal, ${ }^{153}$ T. Lam, ${ }^{153}$ N. Mccoll, ${ }^{153}$ W. A. Nash, ${ }^{153}$ S. Regnard, ${ }^{153}$ D. Saltzberg, ${ }^{153}$ C. Schnaible, ${ }^{153}$ B. Stone, ${ }^{153}$ V. Valuev, ${ }^{153}$ K. Burt, ${ }^{154}$ Y. Chen, ${ }^{154}$ R. Clare, ${ }^{154}$ J. W. Gary, ${ }^{154}$ M. Gordon, ${ }^{154}$ G. Hanson, ${ }^{154}$ G. Karapostoli,${ }^{154}$ O. R. Long, ${ }^{154}$ N. Manganelli, ${ }^{154}$ M. Olmedo Negrete, ${ }^{154}$ W. Si ${ }^{154}$ S. Wimpenny, ${ }^{154}$ Y. Zhang, ${ }^{154}$ J. G. Branson, ${ }^{155}$ P. Chang, ${ }^{155}$ S. Cittolin, ${ }^{155}$ S. Cooperstein,${ }^{155}$ N. Deelen, ${ }^{155}$ J. Duarte, ${ }^{155}$ R. Gerosa ${ }^{155}$ L. Giannini, ${ }^{155}$ D. Gilbert, ${ }^{155}$ J. Guiang, ${ }^{155}$ R. Kansal,${ }^{155}$ V. Krutelyov, ${ }^{155}$ R. Lee, ${ }^{155}$ J. Letts, ${ }^{155}$ M. Masciovecchio, ${ }^{155}$ S. May, ${ }^{155}$ S. Padhi, ${ }^{155}$ M. Pieri, ${ }^{155}$ B. V. Sathia Narayanan, ${ }^{155}$ V. Sharma,,${ }^{155}$ M. Tadel,,${ }^{155}$ A. Vartak,${ }^{155}$ F. Würthwein ${ }^{155}$ Y. Xiang, ${ }^{155}$ A. Yagil, ${ }^{155}$ N. Amin,${ }^{156}$ C. Campagnari, ${ }^{156}$ M. Citron, ${ }^{156}$ A. Dorsett,${ }^{156}$ V. Dutta, ${ }^{156}$ J. Incandela, ${ }^{156}$ M. Kilpatrick, ${ }^{156}$ J. Kim, ${ }^{156}$ B. Marsh, ${ }^{156}$ H. Mei, ${ }^{156}$ M. Oshiro, ${ }^{156}$ A. Ovcharova, ${ }^{156}$ M. Quinnan, ${ }^{156}$ J. Richman, ${ }^{156}$ U. Sarica, ${ }^{156}$ D. Stuart, ${ }^{156}$ S. Wang, ${ }^{156}$ A. Bornheim, ${ }^{157}$ O. Cerri, ${ }^{157}$ I. Dutta, ${ }^{157}$

J. M. Lawhorn, ${ }^{157}$ N. Lu, ${ }^{157}$ J. Mao ${ }^{157}$ H. B. Newman, ${ }^{157}$ J. Ngadiuba,${ }^{157}$ T. Q. Nguyen, ${ }^{157}$ M. Spiropulu, ${ }^{157}$

J. R. Vlimant, ${ }^{157}$ C. Wang, ${ }^{157}$ S. Xie, ${ }^{157}$ Z. Zhang, ${ }^{157}$ R. Y. Zhu, ${ }^{157}$ J. Alison, ${ }^{158}$ M. B. Andrews, ${ }^{158}$ T. Ferguson, ${ }^{158}$ T. Mudholkar, ${ }^{158}$ M. Paulini, ${ }^{158}$ I. Vorobiev, ${ }^{158}$ J. P. Cumalat, ${ }^{159}$ W. T. Ford, ${ }^{159}$ E. MacDonald, ${ }^{159}$ R. Patel, ${ }^{159}$ A. Perloff, ${ }^{159}$ K. Stenson, ${ }^{159}$ K. A. Ulmer, ${ }^{159}$ S. R. Wagner, ${ }^{159}$ J. Alexander, ${ }^{160}$ Y. Cheng, ${ }^{160}$ J. Chu, ${ }^{160}$ D. J. Cranshaw, ${ }^{160}$ K. Mcdermott, ${ }^{160}$ J. Monroy, ${ }^{160}$ J. R. Patterson, ${ }^{160}$ D. Quach, ${ }^{160}$ J. Reichert, ${ }^{160}$ A. Ryd, ${ }^{160}$ W. Sun, ${ }^{160}$ S. M. Tan, ${ }^{160}$ Z. Tao, ${ }^{160}$ J. Thom, ${ }^{160}$ P. Wittich ${ }^{160}$ M. Zientek, ${ }^{160}$ M. Albrow, ${ }^{161}$ M. Alyari, ${ }^{161}$ G. Apollinari, ${ }^{161}$ A. Apresyan, ${ }^{161}$ A. Apyan, ${ }^{161}$ S. Banerjee, ${ }^{161}$ L. A. T. Bauerdick, ${ }^{161}$ A. Beretvas, ${ }^{161}$ D. Berry, ${ }^{161}$ J. Berryhill, ${ }^{161}$ P. C. Bhat, ${ }^{161}$ K. Burkett, ${ }^{161}$ J. N. Butler, ${ }^{161}$ A. Canepa, ${ }^{161}$ G. B. Cerati, ${ }^{161}$ H. W. K. Cheung, ${ }^{161}$ F. Chlebana,${ }^{161}$ M. Cremonesi, ${ }^{161}$ K. F. Di Petrillo, ${ }^{161}$ V. D. Elvira, ${ }^{161}$ J. Freeman, ${ }^{161}$ Z. Gecse ${ }^{161}$ L. Gray, ${ }^{161}$ D. Green, ${ }^{161}$ S. Grünendahl, ${ }^{161}$ O. Gutsche ${ }^{161}$ R. M. Harris, ${ }^{161}$ R. Heller, ${ }^{161}$ T. C. Herwig, ${ }^{161}$ J. Hirschauer, ${ }^{161}$ B. Jayatilaka, ${ }^{161}$ S. Jindariani, ${ }^{161}$ M. Johnson, ${ }^{161}$ U. Joshi, ${ }^{161}$ P. Klabbers, ${ }^{161}$ T. Klijnsma, ${ }^{161}$ B. Klima, ${ }^{161}$ M. J. Kortelainen, ${ }^{161}$ K. H. M. Kwok, ${ }^{161}$ S. Lammel, ${ }^{161}$ D. Lincoln, ${ }^{161}$ R. Lipton, ${ }^{161}$ T. Liu, ${ }^{161}$ J. Lykken, ${ }^{161}$ C. Madrid, ${ }^{161}$

K. Maeshima, ${ }^{161}$ C. Mantilla, ${ }^{161}$ D. Mason, ${ }^{161}$ P. McBride, ${ }^{161}$ P. Merkel,${ }^{161}$ S. Mrenna, ${ }^{161}$ S. Nahn, ${ }^{161}$ V. O’Dell, ${ }^{161}$ V. Papadimitriou, ${ }^{161}$ K. Pedro, ${ }^{161}$ C. Pena, ${ }^{161, f f f}$ O. Prokofyev, ${ }^{161}$ F. Ravera ${ }^{161}$ A. Reinsvold Hall, ${ }^{161}$ L. Ristori, ${ }^{161}$ B. Schneider, ${ }^{161}$ E. Sexton-Kennedy, ${ }^{161}$ N. Smith, ${ }^{161}$ A. Soha,${ }^{161}$ L. Spiegel,${ }^{161}$ S. Stoynev, ${ }^{161}$ J. Strait, ${ }^{161}$ L. Taylor, ${ }^{161}$ S. Tkaczyk, ${ }^{161}$ N. V. Tran, ${ }^{161}$ L. Uplegger,${ }^{161}$ E. W. Vaandering, ${ }^{161}$ H. A. Weber ${ }^{161}$ A. Woodard,${ }^{161}$ D. Acosta, ${ }^{162}$ P. Avery, ${ }^{162}$ D. Bourilkov, ${ }^{162}$ L. Cadamuro, ${ }^{162}$ V. Cherepanov, ${ }^{162}$ F. Errico, ${ }^{162}$ R. D. Field, ${ }^{162}$ D. Guerrero, ${ }^{162}$ B. M. Joshi, ${ }^{162}$ M. Kim, ${ }^{162}$ J. Konigsberg, ${ }^{162}$ A. Korytov, ${ }^{162}$ K. H. Lo, ${ }^{162}$ K. Matchev, ${ }^{162}$ N. Menendez, ${ }^{162}$ G. Mitselmakher, ${ }^{162}$ D. Rosenzweig, ${ }^{162}$ S. Rosenzweig, ${ }^{162}$ K. Shi, ${ }^{162}$ J. Sturdy, ${ }^{162}$ J. Wang, ${ }^{162}$ E. Yigitbasi, ${ }^{162}$ X. Zuo, ${ }^{162}$ T. Adams, ${ }^{163}$ A. Askew, ${ }^{163}$ D. Diaz, ${ }^{163}$ R. Habibullah, ${ }^{163}$ S. Hagopian, ${ }^{163}$ V. Hagopian, ${ }^{163}$ K. F. Johnson, ${ }^{163}$ R. Khurana, ${ }^{163}$ T. Kolberg, ${ }^{163}$ G. Martinez, ${ }^{163}$

H. Prosper, ${ }^{163}$ C. Schiber, ${ }^{163}$ R. Yohay, ${ }^{163}$ J. Zhang, ${ }^{163}$ M. M. Baarmand, ${ }^{164}$ S. Butalla, ${ }^{164}$ T. Elkafrawy, ${ }^{164, o}$

M. Hohlmann, ${ }^{164}$ R. Kumar Verma, ${ }^{164}$ D. Noonan, ${ }^{164}$ M. Rahmani, ${ }^{164}$ M. Saunders, ${ }^{164}$ F. Yumiceva, ${ }^{164}$ M. R. Adams, ${ }^{165}$ L. Apanasevich, ${ }^{165}$ H. Becerril Gonzalez, ${ }^{165}$ R. Cavanaugh, ${ }^{165}$ X. Chen, ${ }^{165}$ S. Dittmer, ${ }^{165}$ O. Evdokimov, ${ }^{165}$ C. E. Gerber, ${ }^{165}$ 
D. A. Hangal, ${ }^{165}$ D. J. Hofman, ${ }^{165}$ C. Mills, ${ }^{165}$ G. Oh, ${ }^{165}$ T. Roy, ${ }^{165}$ M. B. Tonjes, ${ }^{165}$ N. Varelas, ${ }^{165}$ J. Viinikainen, ${ }^{165}$ X. Wang, ${ }^{165}$ Z. Wu, ${ }^{165}$ Z. Ye, ${ }^{165}$ M. Alhusseini, ${ }^{166}$ K. Dilsiz, ${ }^{166, m m m m}$ S. Durgut, ${ }^{166}$ R. P. Gandrajula, ${ }^{166}$ M. Haytmyradov, ${ }^{166}$ V. Khristenko, ${ }^{166}$ O. K. Köseyan, ${ }^{166}$ J.-P. Merlo, ${ }^{166}$ A. Mestvirishvili, ${ }^{166, n n n n}$ A. Moeller, ${ }^{166}$ J. Nachtman, ${ }^{166}$ H. Ogul, ${ }^{166,0000}$ Y. Onel, ${ }^{166}$ F. Ozok, ${ }^{166, p p p p}$ A. Penzo, ${ }^{166}$ C. Snyder ${ }^{166}$ E. Tiras, ${ }^{166, q q q q}$ J. Wetzel, ${ }^{166}$ O. Amram, ${ }^{167}$ B. Blumenfeld, ${ }^{167}$ L. Corcodilos, ${ }^{167}$ J. Davis, ${ }^{167}$ M. Eminizer, ${ }^{167}$ A. V. Gritsan, ${ }^{167}$ S. Kyriacou, ${ }^{167}$ P. Maksimovic, ${ }^{167}$ J. Roskes, ${ }^{167}$ M. Swartz, ${ }^{167}$ T. Á. Vámi ${ }^{167}$ C. Baldenegro Barrera, ${ }^{168}$ P. Baringer, ${ }^{168}$ A. Bean, ${ }^{168}$ A. Bylinkin, ${ }^{168}$ T. Isidori, ${ }^{168}$ S. Khalil, ${ }^{168}$ J. King, ${ }^{168}$ G. Krintiras, ${ }^{168}$ A. Kropivnitskaya, ${ }^{168}$ C. Lindsey, ${ }^{168}$ N. Minafra, ${ }^{168}$ M. Murray, ${ }^{168}$ C. Rogan,${ }^{168}$ C. Royon, ${ }^{168}$ S. Sanders, ${ }^{168}$ E. Schmitz, ${ }^{168}$ J. D. Tapia Takaki, ${ }^{168}$ Q. Wang, ${ }^{168}$ J. Williams, ${ }^{168}$ G. Wilson, ${ }^{168}$ S. Duric, ${ }^{169}$ A. Ivanov, ${ }^{169}$ K. Kaadze, ${ }^{169}$ D. Kim, ${ }^{169}$ Y. Maravin, ${ }^{169}$ T. Mitchell, ${ }^{169}$ A. Modak, ${ }^{169}$ K. Nam, ${ }^{169}$ F. Rebassoo, ${ }^{170}$ D. Wright,${ }^{170}$ E. Adams, ${ }^{171}$ A. Baden, ${ }^{171}$ O. Baron, ${ }^{171}$ A. Belloni, ${ }^{171}$ S. C. Eno, ${ }^{171}$ Y. Feng, ${ }^{171}$ N. J. Hadley, ${ }^{171}$ S. Jabeen, ${ }^{171}$ R. G. Kellogg, ${ }^{171}$ T. Koeth, ${ }^{171}$ A. C. Mignerey, ${ }^{171}$ S. Nabili, ${ }^{171}$ M. Seidel, ${ }^{171}$ A. Skuja, ${ }^{171}$ S. C. Tonwar, ${ }^{171}$ L. Wang, ${ }^{171}$ K. Wong, ${ }^{171}$ D. Abercrombie, ${ }^{172}$ G. Andreassi, ${ }^{172}$ R. Bi, ${ }^{172}$ S. Brandt, ${ }^{172}$ W. Busza, ${ }^{172}$ I. A. Cali,,${ }^{172}$ Y. Chen, ${ }^{172}$ M. D'Alfonso, ${ }^{172}$ G. Gomez Ceballos, ${ }^{172}$ M. Goncharov, ${ }^{172}$ P. Harris, ${ }^{172}$ M. Hu ${ }^{172}$ M. Klute,${ }^{172}$ D. Kovalskyi, ${ }^{172}$ J. Krupa, ${ }^{172}$ Y.-J. Lee, ${ }^{172}$ B. Maier, ${ }^{172}$ A. C. Marini, ${ }^{172}$ C. Mironov, ${ }^{172}$ C. Paus, ${ }^{172}$ D. Rankin, ${ }^{172}$ C. Roland, ${ }^{172}$ G. Roland, ${ }^{172}$ Z. Shi, ${ }^{172}$ G. S. F. Stephans, ${ }^{172}$ K. Tatar, ${ }^{172}$ J. Wang, ${ }^{172}$ Z. Wang, ${ }^{172}$ B. Wyslouch, ${ }^{172}$ R. M. Chatterjee, ${ }^{173}$ A. Evans,${ }^{173}$ P. Hansen,${ }^{173}$ J. Hiltbrand ${ }^{173}$ Sh. Jain, ${ }^{173}$ M. Krohn, ${ }^{173}$ Y. Kubota,${ }^{173}$ Z. Lesko, ${ }^{173}$ J. Mans, ${ }^{173}$ M. Revering, ${ }^{173}$ R. Rusack, ${ }^{173}$ R. Saradhy, ${ }^{173}$ N. Schroeder, ${ }^{173}$ N. Strobbe, ${ }^{173}$ M. A. Wadud, ${ }^{173}$ J. G. Acosta, ${ }^{174}$ S. Oliveros, ${ }^{174}$ K. Bloom, ${ }^{175}$ M. Bryson, ${ }^{175}$ S. Chauhan, ${ }^{175}$ D. R. Claes, ${ }^{175}$ C. Fangmeier, ${ }^{175}$ L. Finco,${ }^{175}$ F. Golf, ${ }^{175}$ J. R. González Fernández, ${ }^{175}$ C. Joo, ${ }^{175}$ I. Kravchenko, ${ }^{175}$ M. Musich, ${ }^{175}$ J. E. Siado, ${ }^{175}$ G. R. Snow, ${ }^{175, a}$ W. Tabb, ${ }^{175}$ F. Yan, ${ }^{175}$ G. Agarwal, ${ }^{176}$ H. Bandyopadhyay, ${ }^{176}$ L. Hay, ${ }^{176}$ I. Iashvili ${ }^{176}$ A. Kharchilava, ${ }^{176}$ C. McLean, ${ }^{176}$ D. Nguyen, ${ }^{176}$ J. Pekkanen, ${ }^{176}$ S. Rappoccio, ${ }^{176}$ A. Williams, ${ }^{176}$

G. Alverson, ${ }^{177}$ E. Barberis, ${ }^{177}$ C. Freer, ${ }^{177}$ Y. Haddad, ${ }^{177}$ A. Hortiangtham, ${ }^{177}$ J. Li, ${ }^{177}$ G. Madigan, ${ }^{177}$ B. Marzocchi, ${ }^{177}$ D. M. Morse, ${ }^{177}$ V. Nguyen, ${ }^{177}$ T. Orimoto, ${ }^{177}$ A. Parker, ${ }^{177}$ L. Skinnari, ${ }^{177}$ A. Tishelman-Charny, ${ }^{177}$ T. Wamorkar, ${ }^{177}$ B. Wang, ${ }^{177}$ A. Wisecarver, ${ }^{177}$ D. Wood, ${ }^{177}$ S. Bhattacharya, ${ }^{178}$ J. Bueghly, ${ }^{178}$ Z. Chen, ${ }^{178}$ A. Gilbert, ${ }^{178}$ T. Gunter, ${ }^{178}$ K. A. Hahn, ${ }^{178}$ N. Odell,,${ }^{178}$ M. H. Schmitt, ${ }^{178}$ K. Sung,,${ }^{178}$ M. Velasco, ${ }^{178}$ R. Band,${ }^{179}$ R. Bucci, ${ }^{179}$ N. Dev, ${ }^{179}$ R. Goldouzian, ${ }^{179}$ M. Hildreth, ${ }^{179}$ K. Hurtado Anampa, ${ }^{179}$ C. Jessop, ${ }^{179}$ K. Lannon, ${ }^{179}$ N. Loukas,${ }^{179}$ N. Marinelli, ${ }^{179}$ I. Mcalister, ${ }^{179}$ F. Meng, ${ }^{179}$ K. Mohrman, ${ }^{179}$ Y. Musienko, ${ }^{179, y y}$ R. Ruchti, ${ }^{179}$ P. Siddireddy, ${ }^{179}$ M. Wayne,${ }^{179}$

A. Wightman, ${ }^{179}$ M. Wolf, ${ }^{179}$ M. Zarucki, ${ }^{179}$ L. Zygala, ${ }^{179}$ B. Bylsma,${ }^{180}$ B. Cardwell ${ }^{180}$ L. S. Durkin, ${ }^{180}$ B. Francis, ${ }^{180}$ C. Hill, ${ }^{180}$ A. Lefeld, ${ }^{180}$ M. Nunez Ornelas, ${ }^{180}$ K. Wei, ${ }^{180}$ B. L. Winer, ${ }^{180}$ B. R. Yates, ${ }^{180}$ F. M. Addesa,${ }^{181}$ B. Bonham, ${ }^{181}$ P. Das, ${ }^{181}$ G. Dezoort, ${ }^{181}$ P. Elmer ${ }^{181}$ A. Frankenthal, ${ }^{181}$ B. Greenberg, ${ }^{181}$ N. Haubrich, ${ }^{181}$ S. Higginbotham, ${ }^{181}$ A. Kalogeropoulos, ${ }^{181}$ G. Kopp, ${ }^{181}$ S. Kwan, ${ }^{181}$ D. Lange, ${ }^{181}$ M. T. Lucchini,${ }^{181}$ D. Marlow, ${ }^{181}$ K. Mei, ${ }^{181}$ I. Ojalvo, ${ }^{181}$ J. Olsen, ${ }^{181}$ C. Palmer, ${ }^{181}$ D. Stickland, ${ }^{181}$ C. Tully, ${ }^{181}$ S. Malik,${ }^{182}$ S. Norberg, ${ }^{182}$ A. S. Bakshi, ${ }^{183}$ V. E. Barnes,${ }^{183}$ R. Chawla, ${ }^{183}$ S. Das, ${ }^{183}$ L. Gutay, ${ }^{183}$ M. Jones, ${ }^{183}$ A. W. Jung, ${ }^{183}$ S. Karmarkar, ${ }^{183}$ M. Liu, ${ }^{183}$ G. Negro, ${ }^{183}$ N. Neumeister, ${ }^{183}$ G. Paspalaki, ${ }^{183}$ C. C. Peng, ${ }^{183}$ S. Piperov, ${ }^{183}$ A. Purohit, ${ }^{183}$ J. F. Schulte, ${ }^{183}$ M. Stojanovic, ${ }^{183, r}$ J. Thieman, ${ }^{183}$ F. Wang, ${ }^{183}$ R. Xiao, ${ }^{183}$ W. Xie, ${ }^{183}$ J. Dolen, ${ }^{184}$ N. Parashar, ${ }^{184}$ A. Baty, ${ }^{185}$ S. Dildick, ${ }^{185}$ K. M. Ecklund ${ }^{185}$ S. Freed, ${ }^{185}$ F. J. M. Geurts, ${ }^{185}$

A. Kumar, ${ }^{185}$ W. Li, ${ }^{185}$ B. P. Padley, ${ }^{185}$ R. Redjimi ${ }^{185}$ J. Roberts, ${ }^{185, a}$ W. Shi ${ }^{185}$ A. G. Stahl Leiton, ${ }^{185}$ A. Bodek, ${ }^{186}$ P. de Barbaro, ${ }^{186}$ R. Demina, ${ }^{186}$ J. L. Dulemba, ${ }^{186}$ C. Fallon, ${ }^{186}$ T. Ferbel, ${ }^{186}$ M. Galanti, ${ }^{186}$ A. Garcia-Bellido, ${ }^{186}$ O. Hindrichs, ${ }^{186}$ A. Khukhunaishvili, ${ }^{186}$ E. Ranken, ${ }^{186}$ R. Taus, ${ }^{186}$ B. Chiarito, ${ }^{187}$ J. P. Chou, ${ }^{187}$ A. Gandrakota, ${ }^{187}$ Y. Gershtein, ${ }^{187}$ E. Halkiadakis, ${ }^{187}$ A. Hart, ${ }^{187}$ M. Heindl, ${ }^{187}$ E. Hughes, ${ }^{187}$ S. Kaplan, ${ }^{187}$ O. Karacheban, ${ }^{187, z}$ I. Laflotte, ${ }^{187}$ A. Lath, ${ }^{187}$ R. Montalvo, ${ }^{187}$ K. Nash, ${ }^{187}$ M. Osherson, ${ }^{187}$ S. Salur, ${ }^{187}$ S. Schnetzer, ${ }^{187}$ S. Somalwar, ${ }^{187}$ R. Stone, ${ }^{187}$ S. A. Thayil, ${ }^{187}$ S. Thomas, ${ }^{187}$ H. Wang ${ }^{187}$ H. Acharya,${ }^{188}$ A. G. Delannoy, ${ }^{188}$ S. Spanier ${ }^{188}$ O. Bouhali, ${ }^{189, r r r r}$ M. Dalchenko, ${ }^{189}$ A. Delgado, ${ }^{189}$ R. Eusebi, ${ }^{189}$ J. Gilmore, ${ }^{189}$ T. Huang, ${ }^{189}$ T. Kamon, ${ }^{189, s s s s}$ H. Kim, ${ }^{189}$ S. Luo, ${ }^{189}$ S. Malhotra, ${ }^{189}$ R. Mueller, ${ }^{189}$ D. Overton, ${ }^{189}$ D. Rathjens, ${ }^{189}$ A. Safonov, ${ }^{189}$ N. Akchurin, ${ }^{190}$ J. Damgov, ${ }^{190}$ V. Hegde, ${ }^{190}$ S. Kunori, ${ }^{190}$ K. Lamichhane, ${ }^{190}$ S. W. Lee, ${ }^{190}$ T. Mengke, ${ }^{190}$ S. Muthumuni, ${ }^{190}$ T. Peltola,${ }^{190}$ S. Undleeb, ${ }^{190}$ I. Volobouev, ${ }^{190}$ Z. Wang, ${ }^{190}$ A. Whitbeck, ${ }^{190}$ E. Appelt ${ }^{191}$ S. Greene, ${ }^{191}$ A. Gurrola ${ }^{191}$ W. Johns, ${ }^{191}$ C. Maguire, ${ }^{191}$ A. Melo, ${ }^{191}$ H. Ni, ${ }^{191}$ K. Padeken, ${ }^{191}$ F. Romeo, ${ }^{191}$ P. Sheldon, ${ }^{191}$ S. Tuo, ${ }^{191}$ J. Velkovska, ${ }^{191}$ M. W. Arenton, ${ }^{192}$ B. Cox,${ }^{192}$ G. Cummings, ${ }^{192}$ J. Hakala, ${ }^{192}$ R. Hirosky, ${ }^{192}$ M. Joyce, ${ }^{192}$ A. Ledovskoy, ${ }^{192}$ A. Li,${ }^{192}$ C. Neu, ${ }^{192}$ B. Tannenwald, ${ }^{192}$ E. Wolfe,${ }^{192}$ P. E. Karchin ${ }^{193}$ N. Poudyal,${ }^{193}$ P. Thapa ${ }^{193}$ K. Black, ${ }^{194}$ T. Bose, ${ }^{194}$ J. Buchanan, ${ }^{194}$ C. Caillol,${ }^{194}$ S. Dasu, ${ }^{194}$ I. De Bruyn, ${ }^{194}$ P. Everaerts, ${ }^{194}$ F. Fienga, ${ }^{194}$ C. Galloni, ${ }^{194}$ H. He, ${ }^{194}$ M. Herndon, ${ }^{194}$ A. Hervé, ${ }^{194}$ U. Hussain, ${ }^{194}$ 
A. Lanaro, ${ }^{194}$ A. Loeliger, ${ }^{194}$ R. Loveless, ${ }^{194}$ J. Madhusudanan Sreekala, ${ }^{194}$ A. Mallampalli, ${ }^{194}$ A. Mohammadi, ${ }^{194}$ D. Pinna,${ }^{194}$ A. Savin, ${ }^{194}$ V. Shang,${ }^{194}$ V. Sharma,${ }^{194}$ W. H. Smith,${ }^{194}$ D. Teague, ${ }^{194}$ S. Trembath-reichert, ${ }^{194}$ and W. Vetens ${ }^{194}$

(CMS Collaboration)

\author{
${ }^{1}$ Yerevan Physics Institute, Yerevan, Armenia \\ ${ }^{2}$ Institut für Hochenergiephysik, Wien, Austria \\ ${ }^{3}$ Institute for Nuclear Problems, Minsk, Belarus \\ ${ }^{4}$ Universiteit Antwerpen, Antwerpen, Belgium \\ ${ }^{5}$ Vrije Universiteit Brussel, Brussel, Belgium \\ ${ }^{6}$ Université Libre de Bruxelles, Bruxelles, Belgium \\ ${ }^{7}$ Ghent University, Ghent, Belgium \\ ${ }^{8}$ Université Catholique de Louvain, Louvain-la-Neuve, Belgium \\ ${ }^{9}$ Centro Brasileiro de Pesquisas Fisicas, Rio de Janeiro, Brazil \\ ${ }^{10}$ Universidade do Estado do Rio de Janeiro, Rio de Janeiro, Brazil \\ ${ }^{11 a}$ Universidade Estadual Paulista, São Paulo, Brazil \\ ${ }^{11 \mathrm{~b}}$ Universidade Federal do ABC, São Paulo, Brazil \\ ${ }^{12}$ Institute for Nuclear Research and Nuclear Energy, Bulgarian Academy of Sciences, Sofia, Bulgaria \\ ${ }^{13}$ University of Sofia, Sofia, Bulgaria \\ ${ }^{14}$ Beihang University, Beijing, China \\ ${ }^{15}$ Department of Physics, Tsinghua University, Beijing, China \\ ${ }^{16}$ Institute of High Energy Physics, Beijing, China \\ ${ }^{17}$ State Key Laboratory of Nuclear Physics and Technology, Peking University, Beijing, China \\ ${ }^{18}$ Sun Yat-Sen University, Guangzhou, China \\ ${ }^{19}$ Institute of Modern Physics and Key Laboratory of Nuclear Physics and Ion-beam Application (MOE)—Fudan University, \\ Shanghai, China \\ ${ }^{20}$ Zhejiang University, Hangzhou, China \\ ${ }^{21}$ Universidad de Los Andes, Bogota, Colombia \\ ${ }^{22}$ Universidad de Antioquia, Medellin, Colombia \\ ${ }^{23}$ University of Split, Faculty of Electrical Engineering, Mechanical Engineering and Naval Architecture, Split, Croatia \\ ${ }^{24}$ University of Split, Faculty of Science, Split, Croatia \\ ${ }^{25}$ Institute Rudjer Boskovic, Zagreb, Croatia \\ ${ }^{26}$ University of Cyprus, Nicosia, Cyprus \\ ${ }^{27}$ Charles University, Prague, Czech Republic \\ ${ }^{28}$ Escuela Politecnica Nacional, Quito, Ecuador \\ ${ }^{29}$ Universidad San Francisco de Quito, Quito, Ecuador \\ ${ }^{30}$ Academy of Scientific Research and Technology of the Arab Republic of Egypt, \\ Egyptian Network of High Energy Physics, Cairo, Egypt \\ ${ }^{31}$ Center for High Energy Physics (CHEP-FU), Fayoum University, El-Fayoum, Egypt \\ ${ }^{32}$ National Institute of Chemical Physics and Biophysics, Tallinn, Estonia \\ ${ }^{33}$ Department of Physics, University of Helsinki, Helsinki, Finland \\ ${ }^{34}$ Helsinki Institute of Physics, Helsinki, Finland \\ ${ }^{35}$ Lappeenranta University of Technology, Lappeenranta, Finland \\ ${ }^{36}$ IRFU, CEA, Université Paris-Saclay, Gif-sur-Yvette, France \\ ${ }^{37}$ Laboratoire Leprince-Ringuet, CNRS/IN2P3, Ecole Polytechnique, Institut Polytechnique de Paris, Palaiseau, France \\ ${ }^{38}$ Université de Strasbourg, CNRS, IPHC UMR 7178, Strasbourg, France \\ ${ }^{39}$ Université de Lyon, Université Claude Bernard Lyon 1, CNRS-IN2P3, Institut de Physique Nucléaire de Lyon, Villeurbanne, France \\ ${ }^{40}$ Georgian Technical University, Tbilisi, Georgia \\ ${ }^{41}$ RWTH Aachen University, I. Physikalisches Institut, Aachen, Germany \\ ${ }^{42}$ RWTH Aachen University, III. Physikalisches Institut A, Aachen, Germany \\ ${ }^{43}$ RWTH Aachen University, III. Physikalisches Institut B, Aachen, Germany \\ ${ }^{44}$ Deutsches Elektronen-Synchrotron, Hamburg, Germany \\ ${ }^{45}$ University of Hamburg, Hamburg, Germany \\ ${ }^{46}$ Karlsruher Institut fuer Technologie, Karlsruhe, Germany \\ ${ }^{47}$ Institute of Nuclear and Particle Physics (INPP), NCSR Demokritos, Aghia Paraskevi, Greece \\ ${ }^{48}$ National and Kapodistrian University of Athens, Athens, Greece \\ ${ }^{49}$ National Technical University of Athens, Athens, Greece \\ ${ }^{50}$ University of Ioánnina, Ioánnina, Greece
}


${ }^{51}$ MTA-ELTE Lendület CMS Particle and Nuclear Physics Group, Eötvös Loránd University, Budapest, Hungary

${ }^{52}$ Wigner Research Centre for Physics, Budapest, Hungary

${ }^{53}$ Institute of Nuclear Research ATOMKI, Debrecen, Hungary

${ }^{54}$ Institute of Physics, University of Debrecen, Debrecen, Hungary

${ }^{55}$ Eszterhazy Karoly University, Karoly Robert Campus, Gyongyos, Hungary

${ }^{56}$ Indian Institute of Science (IISc), Bangalore, India

${ }^{57}$ National Institute of Science Education and Research, HBNI, Bhubaneswar, India

${ }^{58}$ Panjab University, Chandigarh, India

${ }^{59}$ University of Delhi, Delhi, India

${ }^{60}$ Saha Institute of Nuclear Physics, HBNI, Kolkata, India

${ }^{61}$ Indian Institute of Technology Madras, Madras, India

${ }^{62}$ Bhabha Atomic Research Centre, Mumbai, India

${ }^{63}$ Tata Institute of Fundamental Research-A, Mumbai, India

${ }^{64}$ Tata Institute of Fundamental Research-B, Mumbai, India

${ }^{65}$ Indian Institute of Science Education and Research (IISER), Pune, India

${ }^{66}$ Department of Physics, Isfahan University of Technology, Isfahan, Iran

${ }^{67}$ Institute for Research in Fundamental Sciences (IPM), Tehran, Iran

${ }^{68}$ University College Dublin, Dublin, Ireland

${ }^{69 a}$ INFN Sezione di Bari, Bari, Italy

${ }^{69 \mathrm{~b}}$ Università di Bari, Bari, Italy

${ }^{69 \mathrm{c}}$ Politecnico di Bari, Bari, Italy

${ }^{70 a}$ INFN Sezione di Bologna, Bologna, Italy

${ }^{70 \mathrm{~b}}$ Università di Bologna, Bologna, Italy

${ }^{71 a}$ INFN Sezione di Catania, Catania, Italy

${ }^{71 \mathrm{~b}}$ Università di Catania, Catania, Italy

${ }^{72 \mathrm{a}}$ INFN Sezione di Firenze, Firenze, Italy

${ }^{72 \mathrm{~b}}$ Università di Firenze, Firenze, Italy

${ }^{73}$ INFN Laboratori Nazionali di Frascati, Frascati, Italy

${ }^{74 a}$ INFN Sezione di Genova, Genova, Italy

${ }^{74 \mathrm{~b}}$ Università di Genova, Genova, Italy

${ }^{75 a}$ INFN Sezione di Milano-Bicocca, Milano, Italy

${ }^{75 b}$ Università di Milano-Bicocca, Milano, Italy

${ }^{76 a}$ INFN Sezione di Napoli, Napoli, Italy

${ }^{76 \mathrm{~b}}$ Università di Napoli 'Federico II', Napoli, Italy

${ }^{76 c}$ Università della Basilicata, Potenza, Italy

${ }^{76 \mathrm{~d}}$ Università G. Marconi, Roma, Italy

${ }^{77}$ INFN Sezione di Padova, Padova, Italy

${ }^{77 b}$ Università di Padova, Padova, Italy

${ }^{77 \mathrm{c}}$ Università di Trento, Trento, Italy

${ }^{78 a}$ INFN Sezione di Pavia, Bologna, Italy

${ }^{78 b}$ Università di Pavia, Bologna, Italy

${ }^{79 a}$ INFN Sezione di Perugia, Perugia, Italy

${ }^{79 b}$ Università di Perugia, Perugia, Italy

${ }^{80 a}$ INFN Sezione di Pisa, Pisa Italy

${ }^{80 \mathrm{~b}}$ Università di Pisa, Pisa Italy

${ }^{80 c}$ Scuola Normale Superiore di Pisa, Pisa Italy

${ }^{80 \mathrm{~d}}$ Università di Siena, Siena, Italy

${ }^{81 \mathrm{a}}$ INFN Sezione di Roma, Rome, Italy

${ }^{81 \mathrm{~b}}$ Sapienza Università di Roma, Rome, Italy

${ }^{82 \mathrm{a}}$ INFN Sezione di Torino, Torino, Italy

${ }^{82 \mathrm{~b}}$ Università di Torino, Torino, Italy

${ }^{82 \mathrm{c}}$ Università del Piemonte Orientale, Novara, Italy

${ }^{83 a}$ INFN Sezione di Trieste, Trieste, Italy

${ }^{83 \mathrm{~b}}$ Università di Trieste, Trieste, Italy

${ }^{84}$ Kyungpook National University, Daegu, Korea

${ }^{85}$ Chonnam National University, Institute for Universe and Elementary Particles, Kwangju, Korea

${ }^{86}$ Hanyang University, Seoul, Korea

${ }^{87}$ Korea University, Seoul, Korea

${ }^{88}$ Kyung Hee University, Department of Physics, Seoul, Republic of Korea

${ }^{89}$ Sejong University, Seoul, Korea 


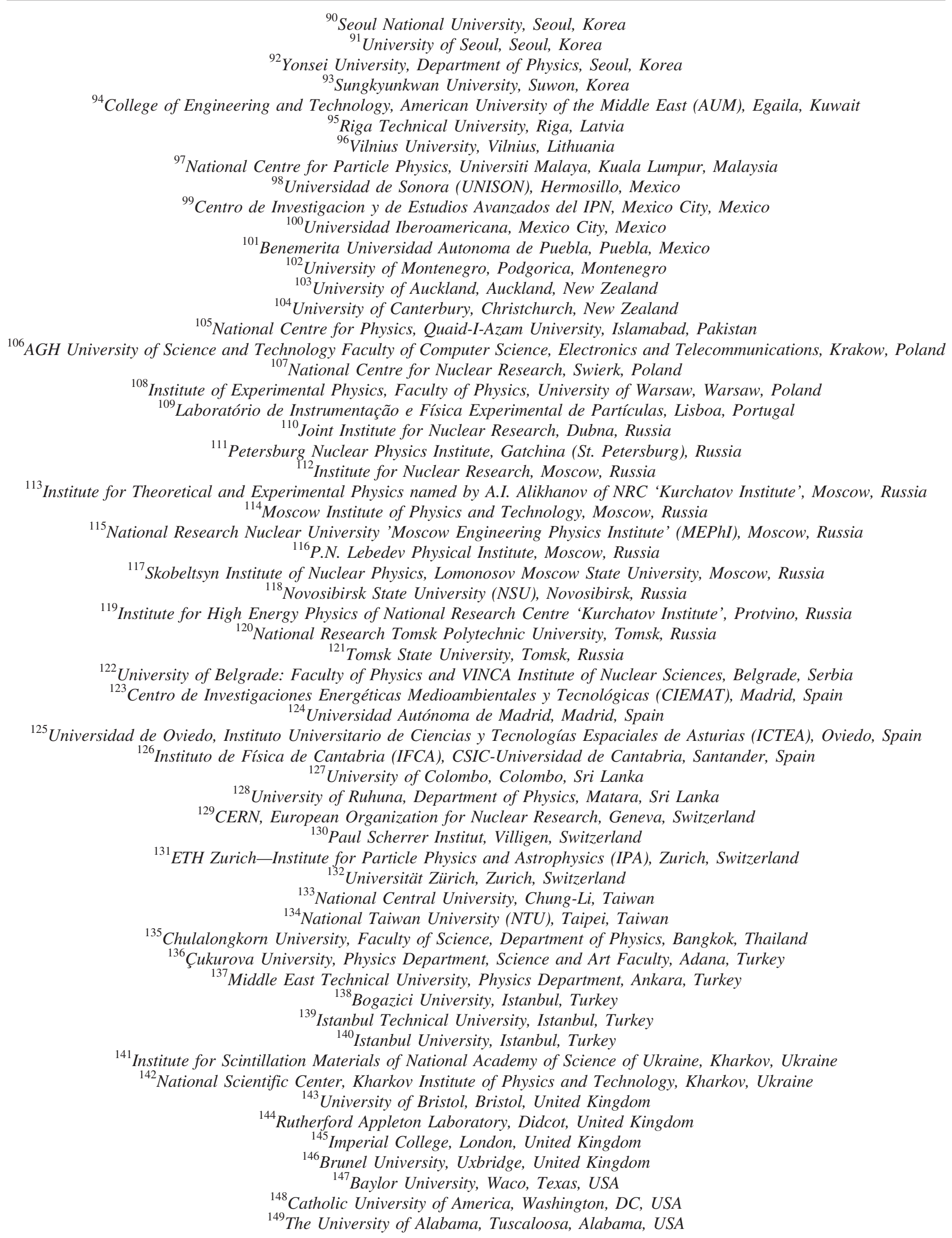




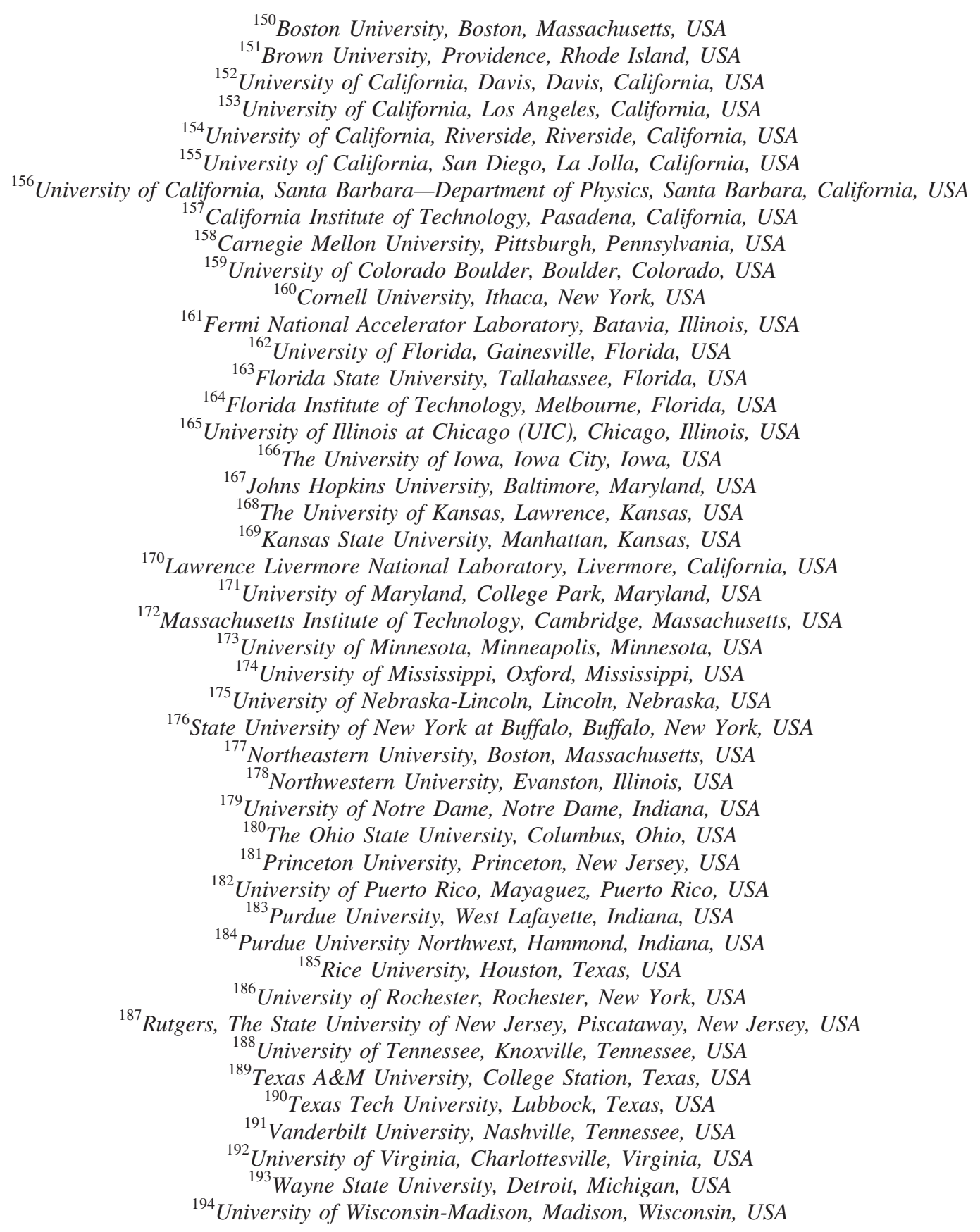

${ }^{\mathrm{a}}$ Deceased.

${ }^{\mathrm{b}}$ Also at Vienna University of Technology, Vienna, Austria.

${ }^{\mathrm{c}}$ Also at Institute of Basic and Applied Sciences, Faculty of Engineering, Arab Academy for Science, Technology and Maritime Transport, Alexandria, Egypt.

${ }^{\mathrm{d}}$ Also at Université Libre de Bruxelles, Bruxelles, Belgium.

${ }^{\mathrm{e}}$ Also at Universidade Estadual de Campinas, Campinas, Brazil.

${ }^{\mathrm{f}}$ Also at Federal University of Rio Grande do Sul, Porto Alegre, Brazil.

${ }^{g}$ Also at University of Chinese Academy of Sciences.

${ }^{\mathrm{h}}$ Also at Department of Physics, Tsinghua University, Beijing, China.

${ }^{i}$ Also at Universidade Federal de Mato Grosso do Sul, Mato Grosso, Brazil.

${ }^{\mathrm{j}}$ Also at The University of Iowa, Iowa City, Iowa, USA.

${ }^{k}$ Also at Nanjing Normal University Department of Physics.

${ }^{1}$ Also at University of Chinese Academy of Sciences, Beijing, China. 
${ }^{\mathrm{m}}$ Also at Institute for Theoretical and Experimental Physics named by A.I. Alikhanov of NRC 'Kurchatov Institute', Moscow, Russia.

${ }^{\mathrm{n}}$ Also at Joint Institute for Nuclear Research, Dubna, Russia.

${ }^{\circ}$ Also at Ain Shams University, Cairo, Egypt.

${ }^{\mathrm{p}}$ Also at Zewail City of Science and Technology, Zewail, Egypt.

${ }^{\mathrm{q}}$ Also at British University in Egypt, Cairo, Egypt.

${ }^{\mathrm{r}}$ Also at Purdue University, West Lafayette, Indiana, USA.

${ }^{\mathrm{s}}$ Also at Université de Haute Alsace, Mulhouse, France.

${ }^{\mathrm{t}}$ Also at Tbilisi State University, Tbilisi, Georgia.

${ }^{\mathrm{u}}$ Also at Erzincan Binali Yildirim University, Erzincan, Turkey.

${ }^{v}$ Also at CERN, European Organization for Nuclear Research, Geneva, Switzerland.

${ }^{w}$ Also at RWTH Aachen University, III. Physikalisches Institut A, Aachen, Germany.

${ }^{x}$ Also at University of Hamburg, Hamburg, Germany.

${ }^{y}$ Also at Department of Physics, Isfahan University of Technology, Isfahan, Iran.

${ }^{\mathrm{z}}$ Also at Brandenburg University of Technology, Cottbus, Germany.

${ }^{\text {aa }}$ Also at Skobeltsyn Institute of Nuclear Physics, Lomonosov Moscow State University, Moscow, Russia.

${ }^{\mathrm{bb}}$ Also at Physics Department, Faculty of Science, Assiut University.

${ }^{c c}$ Also at Eszterhazy Karoly University, Karoly Robert Campus, Gyongyos, Hungary.

${ }^{\mathrm{dd}}$ Also at Institute of Physics, University of Debrecen, Debrecen, Hungary.

${ }^{\mathrm{ee}}$ Also at Institute of Nuclear Research ATOMKI, Debrecen, Hungary.

${ }^{\mathrm{ff}}$ Also at MTA-ELTE Lendület CMS Particle and Nuclear Physics Group, Eötvös Loránd University, Budapest, Hungary.

${ }^{\mathrm{gg}}$ Also at Wigner Research Centre for Physics, Budapest, Hungary.

${ }^{\text {hh }}$ Also at IIT Bhubaneswar, Bhubaneswar, India.

${ }^{\text {ii }}$ Also at Institute of Physics, Bhubaneswar, India.

${ }^{\mathrm{jj}}$ Also at G.H.G. Khalsa College, Punjab, India.

${ }^{\mathrm{kk}}$ Also at Shoolini University, Solan, India.

${ }^{11}$ Also at University of Hyderabad, Hyderabad, India.

${ }^{\mathrm{mm}}$ Also at University of Visva-Bharati, Santiniketan, India.

${ }^{\mathrm{nn}}$ Also at Indian Institute of Technology (IIT), Mumbai, India.

${ }^{\text {oo }}$ Also at Deutsches Elektronen-Synchrotron, Hamburg, Germany.

${ }^{\mathrm{pp}}$ Also at Sharif University of Technology, Tehran, Iran.

${ }^{\mathrm{qq}}$ Also at Department of Physics, University of Science and Technology of Mazandaran, Behshahr, Iran.

${ }^{\mathrm{rr}}$ Also at INFN Sezione di Bari, Università di Bari, Politecnico di Bari, Bari, Italy.

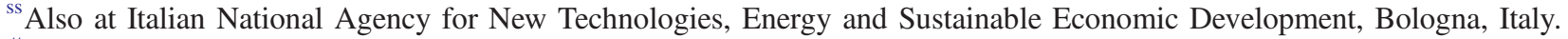

"Also at Centro Siciliano di Fisica Nucleare e di Struttura Della Materia, Catania, Italy.

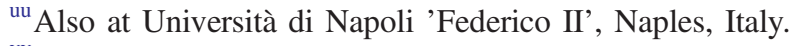

${ }^{\mathrm{v}}$ Also at Riga Technical University, Riga, Latvia.

${ }^{w w}$ Also at Consejo Nacional de Ciencia y Tecnología, Mexico City, Mexico.

${ }^{\mathrm{xx}}$ Also at IRFU, CEA, Université Paris-Saclay, Gif-sur-Yvette, France.

${ }^{\text {yy }}$ Also at Institute for Nuclear Research, Moscow, Russia.

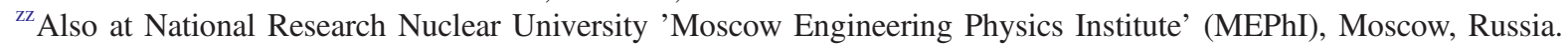

${ }^{\text {aaa }}$ Also at St. Petersburg State Polytechnical University, St. Petersburg, Russia.

${ }^{b b b}$ Also at University of Florida, Gainesville, Florida, USA.

${ }^{c c c}$ Also at Imperial College, London, United Kingdom.

${ }^{\text {ddd }}$ Also at Moscow Institute of Physics and Technology, Moscow, Russia.

${ }^{e e e}$ Also at P.N. Lebedev Physical Institute, Moscow, Russia.

${ }^{\mathrm{fff}}$ Also at California Institute of Technology, Pasadena, California, USA.

${ }^{\text {ggg }}$ Also at Budker Institute of Nuclear Physics, Novosibirsk, Russia.

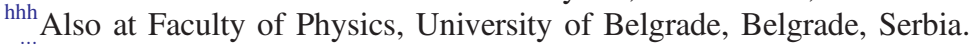

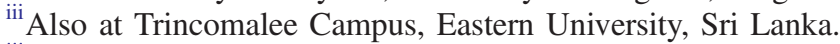

${ }_{\mathrm{jjj}}$ Also at INFN Sezione di Pavia, Università di Pavia, Pavia, Italy.

${ }^{k k k}$ Also at National and Kapodistrian University of Athens, Athens, Greece.

${ }^{111}$ Also at Ecole Polytechnique Fédérale Lausanne, Lausanne, Switzerland.

${ }^{\mathrm{mmm}}$ Also at Universität Zürich, Zurich, Switzerland.

${ }^{n n n}$ Also at Stefan Meyer Institute for Subatomic Physics, Vienna, Austria.

${ }^{\circ o o}$ Also at Laboratoire d'Annecy-le-Vieux de Physique des Particules, IN2P3-CNRS, Annecy-le-Vieux, France.

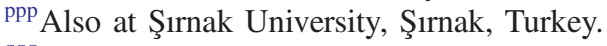

${ }^{\mathrm{qqq}}$ Also at Near East University, Research Center of Experimental Health Science, Nicosia, Turkey.

${ }^{\mathrm{rrr}}$ Also at Konya Technical University, Konya, Turkey.

${ }^{\text {sss }}$ Also at Istanbul University-Cerraphasa, Faculty of Engineering. 


\footnotetext{
${ }^{t t t}$ Also at Mersin University, Mersin, Turkey.

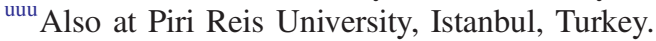

${ }^{\mathrm{vvv}}$ Also at Adiyaman University, Adiyaman, Turkey.

${ }^{w w w}$ Also at Ozyegin University, Istanbul, Turkey.

${ }^{\mathrm{xxx}}$ Also at Izmir Institute of Technology, Izmir, Turkey.

${ }^{y y y}$ Also at Necmettin Erbakan University, Konya, Turkey.

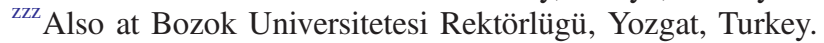

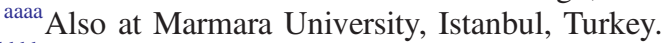

${ }^{b b b b}$ Also at Milli Savunma University, Istanbul, Turkey.

${ }^{c c c c}$ Also at Kafkas University, Kars, Turkey.

${ }^{\text {dddd }}$ Also at Istanbul Bilgi University, Istanbul, Turkey.

${ }^{\text {eeee }}$ Also at Hacettepe University, Ankara, Turkey.

${ }^{\text {ffff }}$ Also at Vrije Universiteit Brussel, Brussel, Belgium.

${ }^{\text {gggg }}$ Also at School of Physics and Astronomy, University of Southampton, Southampton, United Kingdom.

${ }^{\text {hhhh }}$ Also at IPPP Durham University, Durham, United Kingdom.

${ }^{\text {iiii }}$ Also at Monash University, Faculty of Science, Clayton, Australia.

jijj Also at Università di Torino, Torino, Italy.

${ }^{k k k k}$ Also at Bethel University, St. Paul, Minneapolis, USA.

${ }^{1111}$ Also at Karamanoğlu Mehmetbey University, Karaman, Turkey.

${ }^{\mathrm{mmmm}}$ Also at Bingol University, Bingol, Turkey.

${ }^{n n n n}$ Also at Georgian Technical University, Tbilisi, Georgia.

${ }^{0000}$ Also at Sinop University, Sinop, Turkey.

${ }^{\text {pppp }}$ Also at Mimar Sinan University, Istanbul, Istanbul, Turkey.

${ }^{\text {qqqq }}$ Also at Erciyes University, Kaysen, Turkey.

${ }^{\text {rrrr }}$ Also at Texas A\&M University at Qatar, Doha, Qatar.

${ }^{\text {ssss }}$ Also at Kyungpook National University, Daegu, Korea.
} 